\title{
Brain Mediators of Predictive Cue Effects on Perceived Pain
}

\author{
Lauren Y. Atlas, ${ }^{1}$ Niall Bolger, ${ }^{1}$ Martin A. Lindquist, ${ }^{2}$ and Tor D. Wager ${ }^{3}$ \\ Departments of ${ }^{1}$ Psychology and ${ }^{2}$ Statistics, Columbia University, New York, New York 10027, and ${ }^{3}$ Department of Psychology, University of Colorado, \\ Boulder, Colorado 80309
}

Information about upcoming pain strongly influences pain experience in experimental and clinical settings, but little is known about the brain mechanisms that link expectation and experience. To identify the pathways by which informational cues influence perception, analyses must jointly consider both the effects of cues on brain responses and the relationship between brain responses and changes in reported experience. Our task and analysis strategy were designed to test these relationships. Auditory cues elicited expectations for barely painful or highly painful thermal stimulation, and we assessed how cues influenced human subjects' pain reports and brain responses to matched levels of noxious heat using functional magnetic resonance imaging. We used multilevel mediation analysis to identify brain regions that (1) are modulated by predictive cues, (2) predict trial-to-trial variations in pain reports, and (3) formally mediate the relationship between cues and reported pain. Cues influenced heat-evoked responses in most canonical pain-processing regions, including both medial and lateral pain pathways. Effects on several regions correlated with pretask expectations, suggesting that expectancy plays a prominent role. A subset of pain-processing regions, including anterior cingulate cortex, anterior insula, and thalamus, formally mediated cue effects on pain. Effects on these regions were in turn mediated by cue-evoked anticipatory activity in the medial orbitofrontal cortex $(\mathrm{OFC})$ and ventral striatum, areas not previously directly implicated in nociception. These results suggest that activity in pain-processing regions reflects a combination of nociceptive input and top-down information related to expectations, and that anticipatory processes in OFC and striatum may play a key role in modulating pain processing.

\section{Introduction}

Pain has traditionally been considered a veridical perception of the body state. Current accounts present a dramatically different view: They suggest that pain is modifiable by expectations and other cognitive and affective processes (Bingel et al., 2007; Bingel and Tracey, 2008). But what is modified-how nociceptive signals are processed and pain is experienced, or merely how pain is remembered and reported (Godinho et al., 2006)? If expectations influence nociception, then two predictions follow. First, cues that elicit expectations should influence responses to noxious events in the pain processing network (PPN), the set of brain regions that responds most reliably to changes in noxious input (Treede et al., 1999; Peyron et al., 2000; Apkarian et al., 2005). There is mounting evidence that this is the case (Wager et al., 2004; Koyama et al., 2005; Lorenz et al., 2005; Price et al., 2007). Second, cue-induced changes in the PPN should formally mediate cue effects on reported pain. To our knowledge, this prediction has never been tested.

Received Jan. 6, 2010; revised June 23, 2010; accepted July 31, 2010.

This paper was made possible with the support of funding from National Science Foundation Grant 0631637 and National Institutes of Health Grants R01 MH076136 and R01 DA027794 and support from the Gatsby Initiative in Brain Circuitry (T.D.W.) Development of the spiral in-out pulse sequence was funded by P41 RR009784 (principal investigator: Gary Glover). We thank Kate Dahl, Stephen Dashnaw, Joy Hirsch, and Gary Glover for their invaluable contributions to this work, and Ethan Kross and Damon Abraham for collecting data used to localize the painprocessing network in this study. We also thank Mathieu Roy, Tal Yarkoni, Edward E. Smith, F. Caroline Davis, and Gregory R. Samanez-Larkin for feedback on various stages of this manuscript.

Correspondence should be addressed to Lauren Y. Atlas, Department of Psychology, Columbia University, 406 Schermerhorn Hall, 1190 Amsterdam Avenue, Mail Code 5501, New York, NY 10027. E-mail: latlas@psych. columbia.edu.

DOI:10.1523/JNEUROSCI.0057-10.2010

Copyright $\odot 2010$ the authors $\quad 0270-6474 / 10 / 3012964-14 \$ 15.00 / 0$
Testing this mediational link is critical. Modulation of PPN regions, particularly the anterior cingulate and insula, can occur in the absence of physical pain (Singer et al., 2004; Moulton et al., 2005; Zaki et al., 2007), and judgments about pain might be influenced in ways unrelated to nociceptive processes. Therefore, predictive cues may affect PPN activity, but these effects need not mediate changes in pain experience. Thus, the issue of whether expectations influence nociceptive processing remains unresolved. Our primary goal was to test whether PPN activity formally mediates the effects of experimentally manipulated cues on reported pain.

If PPN modulations shape pain experience, a second fundamental question is how those changes come about. Previous research has implicated two systems in the generation of cue-based expectancies: a dorsolateral frontoparietal system implicated in cognitive control and working memory (Corbetta and Shulman, 2002) and a "limbic" circuit, including orbitofrontal cortex, ventral striatum, and amygdala, thought to play a central role in affective processing and value-driven learning (Pessoa, 2009). However, the hypothesis that activity in these systems mediates cue effects on PPN activity has not been tested.

We used multilevel mediation, a recently developed path analysis method (Kenny et al., 2003; Lim et al., 2009; Wager et al., 2009), to link experimentally manipulated expectancy directly to trial-by-trial variations in brain activity and pain reports within subjects. Conditioned auditory cues elicited expectations for low or high painful thermal stimulation, and we assessed cue effects within a single level of noxious heat (Fig. 1). In analysis 1, predictive cues, trial-by-trial measures of voxelwise brain responses, and reported pain were related using the path model shown in 


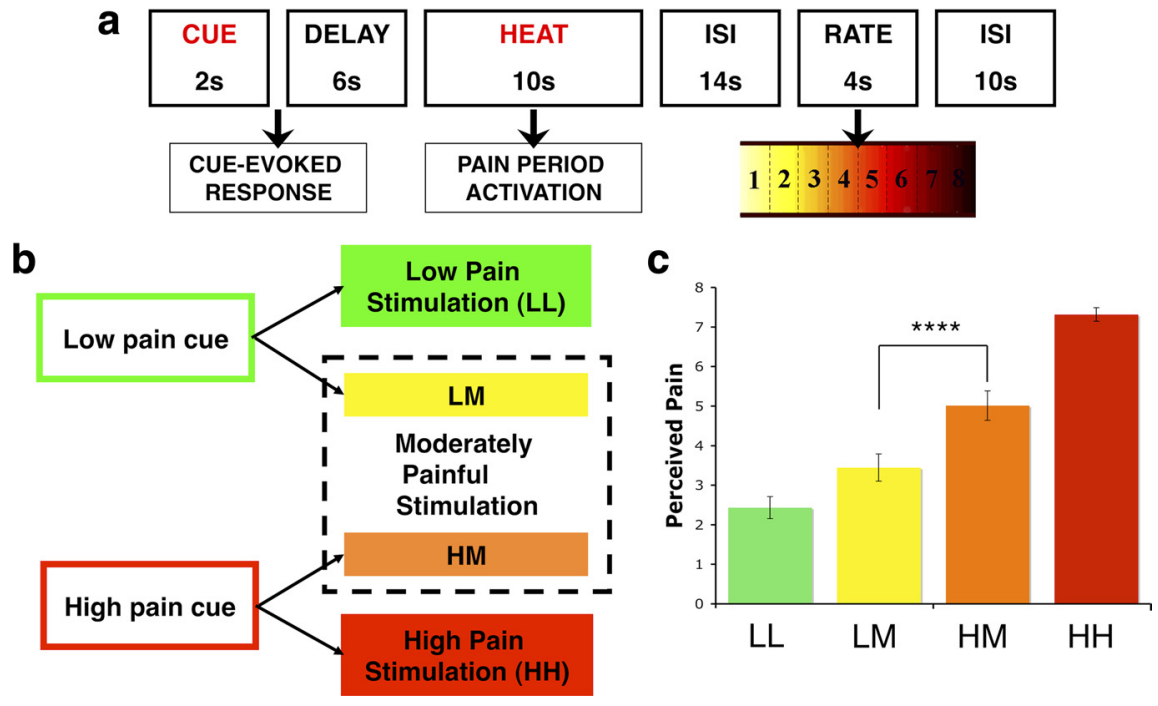

Figure 1. Experimental design and behavioral results. $\boldsymbol{a}$, Trial structure. Each trial consisted of an auditory predictive cue followed by an anticipatory delay and $10 \mathrm{~s}$ of noxious thermal stimulation. $\mathrm{fMRI}$ analyses used single trial analysis to model brain responses evoked during anticipation and noxious thermal stimulation periods. After a fixed interstimulus interval, participants reported trial-by-trial perceived pain using a visual analog scale. $\boldsymbol{b}$, Conditions of interest. During the first two runs of the task, low-pain cues always preceded low-pain stimulation (LL) and high-pain cues preceded high-pain stimulation (HH). In runs three through eight, trials were evenly divided between these conditions and trials in which each predictive cue was followed by a stimulus calibrated to elicit moderate pain [high cue plus medium pain (HM); low cue plus medium pain (LM)]. We included only HM and LM trials in our mediation analyses to examine cue-based expectancy effects during a single level of noxious thermal stimulation. c, Behavioral results. All participants reported greater pain on HM than LM trials. This difference comprises the "total effect" (path c) in our pain-period multilevel mediation analysis (analysis 1$) .{ }^{* * *} p<0.0001$.

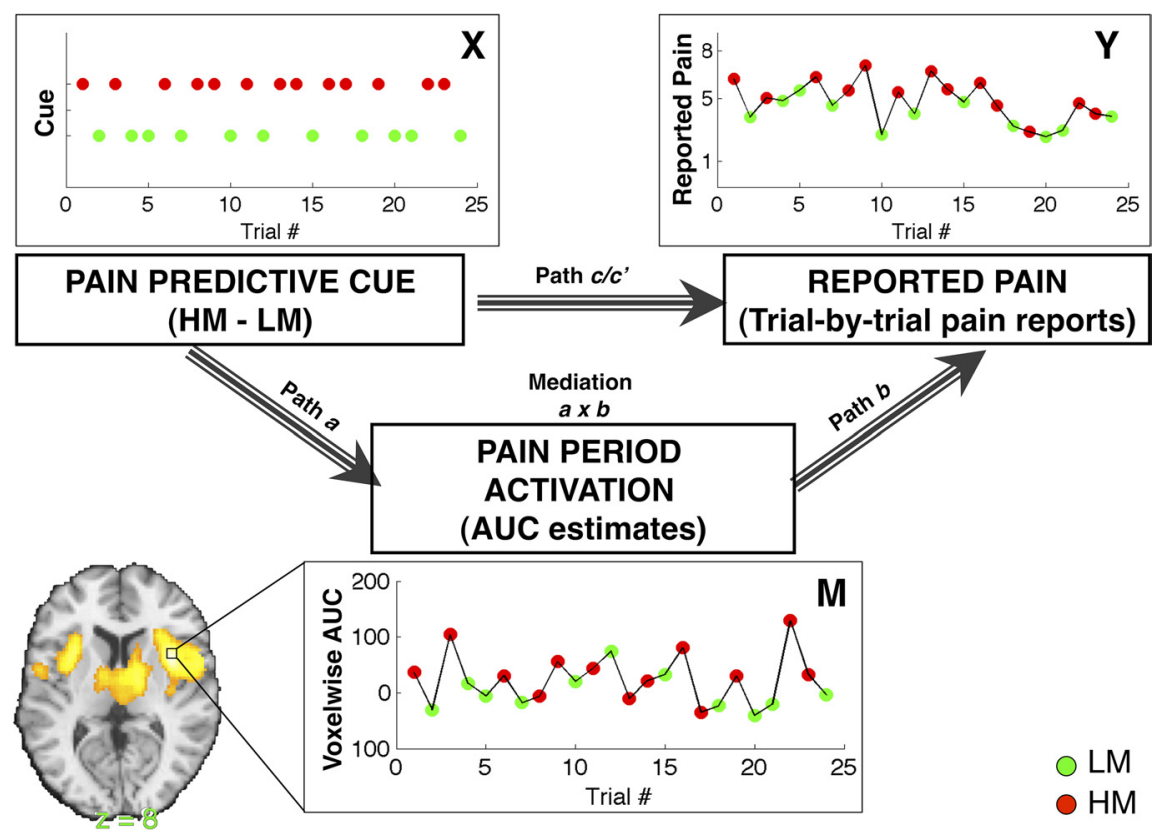

Figure 2. Mediation hypothesis framework: analysis 1 . Our primary analysis examines the dynamic relationships between pain predictive cue [top left; $X$; green, low cue plus medium pain (LM); red, high cue plus medium pain (HM)], voxelwise pain-evoked responses (bottom, $\mathrm{M}$; single trial analysis estimates of $\mathrm{AUC}$, the area under the curve), and trial-by-trial pain reports (top right; $\mathrm{Y}$ ). The four components of multilevel mediation analysis address the current study's key questions. Top, Path $c / c^{\prime}$ : Do predictive cues affect perceived pain as measured by trial-by-trial pain reports? Path c reflects the total relationship between predictive cue and reported pain on medium trials, and path $c^{\prime}$ reflects the direct behavioral relationship, controlling for activity in the mediator - in this case a brain voxel or region. Left, Path $a$ ("cue effects") provides inferences on whether brain voxels are modulated by predictive cue during a constant level of noxious thermal stimulation (Fig. 3, Fig. S2, available at www.jneurosci.org as supplemental material). Right, Path $b$ ("reportrelated responses") provides inferences on whether brain activity in each voxel predicts trial-by-trial pain reports, controlling for cue (stimulus temperature was held constant) (Fig. 4, Fig. S3, available at www.jneurosci.org as supplemental material). Middle, The $a \times b$ mediation effect provides inferences on whether brain voxels explain a significant amount of the covariance between predictive cues and perceived pain (Fig. 5, Fig. S4, available at www.jneurosci.org as supplemental material).
Figure 2. We tested the prediction that PPN regions mediate predictive cue effects on reported experience and tested whether individual differences in conscious expectations predicted these effects. Analysis 2 localized regions in which cue-evoked anticipatory activity mediated cue effects on PPN mediators and thus tested the prediction that circuits associated with cognitive control and value learning mediated cue effects on the PPN.

\section{Materials and Methods}

\section{Participants}

Nineteen healthy, right-handed adults $(M$ age $=25.5$ years, nine female) completed the functional magnetic resonance imaging (fMRI) session and provided informed consent in accordance with the Declaration of Helsinki, as approved by the Columbia University Institutional Review Board (New York, NY). Preliminary eligibility was assessed with a general health questionnaire, a pain safety screening form, and an fMRI safety screening form. Subjects reported no history of psychiatric, neurological, or pain disorders. Study eligibility was then determined during a preliminary thermal pain session visit; eligible participants were required to have maximum tolerable pain levels fall within the range of $42-48^{\circ} \mathrm{C}$ and reliable relationships between stimulus temperature and reported pain (minimum $R^{2} \geq 0.40$; see below, Pain calibration procedure). One participant was unable to differentiate between the two tones used in the paradigm during the training task (see below, Training task), and therefore did not complete the fMRI session.

\section{Materials and procedures}

Thermal stimulation and pain ratings. Thermal stimulation was delivered to the volar surface of the left (nondominant) inner forearm using a $16 \times 16 \mathrm{~mm}$ Peltier thermode (Medoc). Each stimulus lasted $10 \mathrm{~s}$, with $1.5 \mathrm{~s}$ ramp-up and ramp-down periods and $7 \mathrm{~s}$ at target temperature. Temperatures were individually calibrated for each participant. Participants rated stimulation on a continuous scale from 0 to 8 ( 0 , no sensation; 1 , nonpainful warmth; 2 , low pain; 5 , moderate pain; 8 , maximum tolerable pain).

Pain calibration procedure. During a preliminary eligibility session and again on the day of scanning, we performed a pain calibration procedure using eight sites on the volar surface of the left forearm. An adaptive staircase procedure was employed to do the following: (1) identify sites on the forearm with similar nociceptive profiles; 2) determine temperatures required to elicit low pain, moderate pain, and high pain; and (3) derive the individual participant's dose-response curve for the relationship between applied thermal stimulation and reported pain (slope, intercept, $R^{2}$ ). Participants delivered verbal ratings on each trial, and an overall rating-by-temperature curve was fitted over the course of this procedure for each participant. For all participants, the first three 
temperatures applied were 41,44 , and $47^{\circ} \mathrm{C}$; this gave us an initial linear rating-by-temperature curve. This initial linear function allowed us to select temperatures predicted to elicit ratings of 2 (low pain), 5 (moderate pain), and 8 (maximum tolerable pain), and we used elicited ratings to iteratively fit this linear function after each trial using linear regression. Upon completion of the procedure (24 pseudorandom trials; one low, one moderate, one maximum tolerable stimulation per skin site), average residuals were calculated for each skin site, and the four sites with the lowest average residuals were used during test. For each subject, the linear function fit over the course of the calibration was used to determine appropriate temperatures for level 2 (low pain; $M=41.1^{\circ} \mathrm{C}, \mathrm{SD}=2.5$ ), level 5 (medium pain; $M=44.2^{\circ} \mathrm{C}, \mathrm{SD}=2.1$ ), and level 8 (high pain; $\left.M=47.1^{\circ} \mathrm{C}, \mathrm{SD}=1.4\right)$ to be applied during the experimental protocol.

Training task. Participants first completed a training task of 20 trials in which they had to discriminate between two tones that they were told would predict low and high painful stimulation, respectively (predictive cues). Tones lasted $2 \mathrm{~s}$ and were either 500 or $1000 \mathrm{~Hz}$, counterbalanced across subjects. Predictive cues were presented in random order, and participants used the left mouse button to identify low-pain cues and the right mouse button to identify high-pain cues. Participants were required to successfully identify at least $90 \%$ of trials to proceed to the fMRI portion of the experiment (one participant was excluded).

Expectancy ratings. Before each run of the fMRI task, subjects heard the low- and high-pain cues and were asked, after each tone, "When you hear this tone, how much pain do you expect?" The first such rating was made before any pairing between predictive cue and noxious stimulation, thus providing a measure of cue effects on pain expectancy uncontaminated by associative learning. Our analyses of expectancy were based on [highpain cue - low-pain cue] differences in this initial rating. The remaining seven ratings served as a manipulation check, allowing us to measure conscious cue-related expectancies and to test whether expectancies changed over the course of the experiment.

fMRI task design. After calibration and training, fMRI images were acquired during eight functional runs (eight trials/run, 64 trials). The thermode was placed on a different skin site for each run, with two total runs per skin site.

On each trial, a $2 \mathrm{~s}$ predictive cue was followed by a $6 \mathrm{~s}$ anticipatory interval during which a fixation cross was presented on the screen (Fig. 1). Thermal stimulation was then delivered via the thermode for $10 \mathrm{~s}(1.5$ $\mathrm{s}$ ramp up from baseline $\left(32^{\circ} \mathrm{C}\right), 7 \mathrm{~s}$ at peak destination temperature, $1.5 \mathrm{~s}$ return to baseline) at levels calibrated to elicit ratings of high, medium, or low pain in pseudorandom order. Following thermal stimulation, a fixation cross was presented for a $14 \mathrm{~s}$ fixed interstimulus interval (ISI). The words "How painful?" then appeared on the screen for $4 \mathrm{~s}$ above an eight-point visual analog scale (VAS). Participants rated the intensity of the preceding stimulus using an fMRI-compatible track ball (Resonance Technologies) with resolution equivalent to the screen resolution (i.e., $\sim 600$ discrete values between 1 and 8 ). The 14 s delay between pain offset and rating was included to maximize our ability to isolate pain period responses; pilot testing demonstrated that there was no difference in the magnitude of cue effects between ratings made immediately after stimulus offset and those made after a delay. Following the rating, a $10 \mathrm{~s}$ ISI concluded the trial.

There were four types of trials. On low cue plus low pain (LL) trials, low-pain cues were followed by slightly painful stimulation (level 2 based on the pain calibration procedure). On high cue plus high pain ( $\mathrm{HH})$ trials, high-pain cues were followed by highly painful stimulation (level 8 ). On low cue plus medium pain (LM) trials, low-pain cues were followed by moderately painful stimulation (level 5). Finally, on high cue plus medium pain (HM) trials, high-pain cues were followed by moderately painful stimulation (level 5). Thus, the temperatures administered in the critical LM and HM trials were identical, but cue-related expectations were that pain would either be higher or lower than what was actually administered; importantly, the discrepancy between expected and actual stimulus intensity was equal for HM and LM trials, but opposite in sign.

Participants first experienced two runs of trials evenly divided between $\mathrm{LL}$ and $\mathrm{HH}$ trials, in pseudorandom order. This phase served primarily to establish cue-outcome relationships. These were followed by six runs of trials equally divided between LL, HH, LM, and HM trials. The order of conditions was pseudorandomized so that each trial occurred at each position within a run, and runs were counterbalanced across subjects using a Latin Square design, with the stipulation that the first two trials of Run 3 be either LL or HH trials. To optimize the likelihood of observing cue-based expectancy effects within the critical medium trials, participants were never informed that medium stimulation would be delivered.

In this report, we examined brain responses evoked during the $10 \mathrm{~s}$ thermal stimulation period and the $8 \mathrm{~s}$ anticipatory period. The model used to quantify these responses is described in detail below. Contrasts between $\mathrm{HH}$ and LL trials examined activity across all runs (1-8), while multilevel mediation analyses of pain-evoked and cue-evoked responses (analysis 1 and analysis 2), which focused on HM and LM trials, were conducted on trials from runs $3-8$. Trials on which subjects failed to register a rating were excluded from both behavioral and imaging analyses $(M=0.67, S D=0.9)$.

Behavioral analysis. Behavioral data were analyzed using custom Matlab software (MathWorks) to implement a linear mixed-effects model and verified using the mixed procedure in SAS 9.1 (SAS Institute). At the first level of the multilevel model, regression coefficients for the effects of stimulation temperature, cue type ( $\mathrm{H}$ vs $\mathrm{L}$ ), and their interaction on pain reports within subjects were estimated for each individual. The secondlevel of the mixed-effects model assessed the significance of these coefficients across individuals, treating the participant as a random variable. The same approach was used to examine the temporal dynamics of cue effects on pain reports, with additional first level regressors for time and the interaction between time and cue type.

\section{fMRI acquisition and preprocessing}

Data acquisition. Whole-brain fMRI data were acquired on a 1.5T GE Signa TwinSpeed Excite HD scanner (GE Medical Systems) at the Functional MRI Research Center at Columbia University. Functional images were acquired with a $\mathrm{T} 2{ }^{*}$-weighted, two-dimensional gradient echo spiral in/out pulse sequence (Glover and Law, 2001) [repitition time $(\mathrm{TR})=$ $2000 \mathrm{~ms}$; echo time $=40 \mathrm{~ms}$; flip angle $=84^{\circ}$; field of view $=224 \mathrm{~mm}$; $64 \times 64$ matrix, $3.5 \times 3.5 \times 4.55 \mathrm{~mm}$ voxels, 24 slices]. Each run lasted 6 $\mathrm{min}$ and $18 \mathrm{~s}$ (189 TRs). Stimulus presentation and data acquisition were controlled using E-Prime software (Psychology Software Tools). Responses were made with the right hand via an MRI-compatible track ball (Resonance Technologies). Visual stimuli were presented through goggles positioned upon the scanner head coil (Avotech), which allowed us to measure in-scanner eye position and pupil dilation (collected using systems from SensoMotoric Instruments). Throughout scanning, we also continuously monitored heart rate (electrocardiogram and peripheral pulse; In vivo Magnitude), skin conductance, and respiration (James Long Company), which were collected using an ADInstruments PowerLab (model 16SP). Only behavioral and brain measures were included in the analysis presented here.

Preprocessing. Functional images were slice acquisition timing and motion corrected using the FMRIB Software Library (http://www.fmrib.ox.ac.uk/ fsl/), Oxford Centre for Functional MRI of the Brain (FMRIB), Oxford, UK. Structural T1-weighted images were coregistered to the first functional image for each subject using an iterative procedure of automated registration with mutual information coregistration in SPM5 (Wellcome Trust Centre for Neuroimaging, London, UK) and manual adjustment of the automated algorithm's starting point until the automated procedure provided satisfactory alignment. Data were smoothed with an $8 \mathrm{~mm}$ full width at halfmaximum Gaussian smoothing kernel using SPM5.

Structural images were normalized to Montreal Neurological Institute (MNI) space using a genetic algorithm (GA)-based normalization. This approach, based on a similar implementation by Wager and Nichols (2003), is a refinement of the standard SPM5 warping and improves intersubject registration. Structural images were first segmented to gray and white matter and spatially normalized to a standard template brain (the MNI avg152T1.img) using SPM5's iterative segmentation/normalization algorithm (Ashburner and Friston, 2005) with default options ( $7 \times 8 \times 7$ nonlinear basis functions). As the standard MNI template brain has a relatively low spatial resolution, we used the SPM5 normalization solution as the starting point for additional refinement using the GA. A study-specific, higher resolution template aligned with MNI space 
was created by averaging the normalized SPM5-warped T1 images. We then used a GA to renormalize each subject's T1 to the group average template. We assessed both correlation with the SPM5 "avg152T1.nii" template and mutual information between subjects and found that the warping is a significant advance on the quality of SPM5's default normalization algorithm. The results showed better gray matter/white matter separation and better gray matter/CSF separation in the group anatomical image. Because the algorithm starts with a group mean warped to MNI space, the resulting images were still registered with MNI space. Normalized functional images were interpolated to $2 \times 2 \times 2 \mathrm{~mm}$ voxels.

Intensity processing localizer. We used an independent localizer to identify brain regions affected by intensity independently of cue-based expectancies. PPN regions of interest were localized in a separate group of individuals $(n=75)$ drawn from three separate thermal pain studies that contrasted high (level 8) versus low (level 2) stimulation in the absence of pain-predictive cues. All participants received the same thermal pain calibration as in the main experimental group, and stimulation consisted of $10-15 \mathrm{~s}$ epochs of heat on the left volar forearm, as in the main experimental group. Individual contrasts between high and low painful stimulation were transformed to $z$-scores within study and normalized to MNI space. We included these normalized images in a one-sample $t$ test of [high - low intensity] contrast values across the three different studies. The group model included an intercept and covariate coding for differences between the three studies. Familywise error correction $(p<0.05)$ using Gaussian random fields as implemented in SPM5 was used to identify voxels showing a significant [high - low intensity] effect, which was used to define the PPN (see Figure S1, available at www.jneurosci.org as supplemental material). Anatomical localization was determined based on the LONI Probabilistic Brain Atlas (Shattuck et al., 2008).

\section{fMRI analyses}

In this paper, we focus on three key analyses described in the following sections. First, we identified regions whose responses varied as a function of changes in noxious stimulus intensity; for this analysis, we used the standard general linear model (GLM) to contrast HH trials with LL trials and with HM trials. Next, we identified brain mediators of cue effects on perceived pain (analysis 1) using whole-brain, multilevel mediation. Finally, we used multilevel mediation to identify brain regions whose anticipatory responses mediated cue effects on pain-evoked responses in PPN mediators (analysis 2). For the GLM analyses of planned contrasts, first-level analysis was conducted using the GLM with trial-by-trial activity estimates (see below, Quantification of single-trial response magnitudes). Second level analysis of planned contrasts was performed using robust regression, a technique that increases statistical power and decreases false positive rates in the presence of outliers (Wager et al., 2005). For multilevel mediation analyses, all analyses were conducted on trialby-trial activity estimates across all participants. Participant was modeled as a random effect in all analyses.

Quantification of single-trial response magnitudes. Typical fMRI analyses estimate average brain responses across a number of trials using the GLM. In the present study, we used a single-trial analysis approach (Büchel et al., 2002; Duann et al., 2002) to separately estimate pain-evoked and cue-evoked responses on a trial-by-trial basis. Several papers have demonstrated that single trial analyses are reliable and offer increased sensitivity, especially in modeling responses to pain (Koyama et al., 2003).

We quantified single-trial response magnitudes by constructing a GLM design matrix with separate regressors for each trial, as in Rissman et al. (2004). However, we used a flexible basis set to model each trial, thus allowing the shape of the modeled hemodynamic response function (HRF) to vary across trials and voxels. Pain-evoked and cue-evoked (anticipatory) responses were estimated separately. The pain period basis set consisted of three curves shifted in time and was customized for thermal pain responses based on previous studies (Lindquist et al., 2009). To estimate cue-evoked responses, the pain anticipation period was fit with a canonical HRF, and truncated at $8 \mathrm{~s}$ to ensure that fitted anticipatory responses were not affected by noxious stimulus-evoked activity. This allowed us to examine the relationship between anticipation and pain period responses.

In addition to regressors for stimulus-evoked responses for each trial, each subject's GLM design matrix also included 12 regressors reflecting estimated head movement ( $x, y, z$, roll, pitch, and yaw) and these vectors squared, a discrete cosine transform high-pass filtering matrix $(120 \mathrm{~s}$ cutoff), indicator vectors for the first two images in each run, and indicator vectors for time points estimated as outliers based on analyses of the whole-brain time series blind to task information. The data for each voxel were regressed on this design matrix. For each trial in each voxel, we reconstructed the fitted response and used the area under the curve (AUC) as a summary estimate of trial-level anticipatory or pain-period activity.

One important consideration in using trialwise estimates rather than fixed canonical HRFs is that estimates for a given trial can be strongly affected by acquisition artifacts that occur during that trial (e.g., sudden motion, scanner pulse artifacts, etc.). For this reason, trial-by-trial variance inflation factors (VIFs; a measure of design-induced uncertainty due in this case to colinearity with nuisance regressors) were calculated, and any trials with VIFs that exceeded 2 or whose whole-brain AUC estimates exceeded 3 SDs from the mean were excluded from first-level GLM analysis $(M=1.94)$. Voxelwise trial-by-trial AUC parameters for the remaining trials were then passed into first-level GLM analyses. Thus, for each type of event this procedure yielded a measure of brain response (i.e., AUC) to each trial in each voxel and for each subject. We subsequently analyzed the average differences among trial types and their relationships with reported pain using the general linear model and multilevel path analysis.

Intensity processing analysis. All eligible trials were included in a twolevel GLM to examine whether intensity processing was intact in the context of manipulated cues (HH vs LL; HH vs HM). Four first-level regressors were specified for LL trials, LM trials, HM trials, and HH trials, and then contrasts were computed within subjects and subjected to second-level (group) analysis using robust regression. In each first-level model, regressors of interest were modeled along with separate regressors for each of the eight scanning runs, as well as nuisance regressors reflecting subject motion parameter estimates calculated during motion correction; motion, motion squared, derivatives of motion estimates, and squared derivatives were all included in the GLM.

Whole-brain multilevel mediation. Standard fMRI models, such as those we use to examine intensity processing ( $\mathrm{HH}-\mathrm{LL}$ and $\mathrm{HH}-\mathrm{HM}$, described above) assess the relationship between experimental manipulations and brain activity or between brain activity and behavior. Mediation analyses extend the univariate model by incorporating an additional outcome variable (perceived pain in analysis 1, PPN response in analysis 2). The path model jointly tests three effects that are required if a brain region links predictive cue with the outcome: cue effects on brain activity, the relationship between brain activity and the outcome, and formal mediation effects. Each effect is described in detail below.

In the principal model we present here (analysis 1), the initial variable $(X)$ in the path model is the experimentally manipulated predictive cue (which takes on values of 1 for high-pain cue $[\mathrm{H}]$, and -1 for low-pain cue $[\mathrm{L}]$ ), and the outcome variable $(Y)$ is the subject's series of ratings on medium pain trials (Fig. 2). The total effect of $X$ on $Y$ reflects the observed behavioral effects of predictive cue on reported pain and is referred to as path $c$. The mediating variable $(M)$ is a single voxel's series of AUC estimates during medium pain stimulation. The direct effect of $X$ on $Y$ or the effect of predictive cue on reported pain controlling for the mediator (e.g., voxelwise activity) is referred to as path $c^{\prime}$. In analysis 2 , the initial variable is the same $(X, \mathrm{H}-\mathrm{L}$ cue), but $Y$ is replaced with the series of average pain period AUC estimates for PPN mediator activity. The mediating variable in analysis 2 is each voxel's series of anticipation period AUC estimates. For simplicity, the following descriptions of path effects focus on analysis 1 .

One effect of interest is the effect of predictive cue on brain activity, which we refer to as path $a$ or "cue effects." This is equivalent to the $[\mathrm{HM}-\mathrm{LM}]$ contrast in a standard GLM analysis. The path $a$ effect therefore lines up closely with previous approaches to cue-based pain modulation (e.g., Koyama et al., 2005; Keltner et al., 2006). However, we compared two conditions with the same temperature but equal and opposite deviations from expectation, whereas in previous studies trials on which expectancies were violated (invalid cues) were compared to validly cued trials. Although this means we were not able to compare our expect- 
ancy effects to a validly cued medium intensity stimulus, with our design it was possible to test whether pain was influenced by the valence of participants' expectations, holding constant the degree of ambiguity.

A second effect of interest is the association between brain activity $(M)$ and reported pain $(Y)$, which we refer to as path $b$ or "report-related responses." As is standard in directed path models, path $b$ is assessed while controlling for $X(\mathrm{H}-\mathrm{L}$ cue $)$ so that significant relationships between brain activity and pain are assessed controlling for the experimental task manipulation. This means that path $a$ and path $b$ are uncorrelated under the null hypothesis of no mediation and that they identify two separable processes that are likely to contribute to cue-based modulation of perceived pain: (1) the effect of predictive cues on pain-evoked responses (path $a$ ); and (2) the relationship between pain-evoked responses and subjective pain (path $b$ ). The path $b$ effect thereby identifies regions that predict trial-to-trial variations in reported pain, controlling for both cue and temperature-in other words, regions that predict endogenously driven variations in pain. This parametric approach is related to recent approaches to studying pain (Coghill et al., 1999; Craig et al., 2000; Bornhövd et al., 2002; Büchel et al., 2002; Mohr et al., 2005; Wiech et al., 2006; Rolls et al., 2008), economic value (Hare et al., 2008; Plassmann et al., 2008; Chib et al., 2009), and other applications that identify brain regions that predict parametric variations in behavior on a withinsubjects basis. However, our path $b$ effects provide an additional level of specificity, identifying voxels that predict variability controlling for differences associated with task effects (controlling for $X$ ). The path $b$ test can therefore identify regions that contribute to subjective pain reports that may have little, if anything, to do with either nociception (such as those involved in decision making and evaluative judgments) or with cues. Thus, this effect provides complementary yet quite distinct information to traditional parametric analysis or conjunctions across parametric regressors and is closely aligned with recent work examining relationships between prestimulus fluctuations in ongoing brain activity and variability in reported pain (Boly et al., 2007; Ploner et al., 2010). We note that this component of the path model is essentially correlational and, unlike with path $a$, we cannot make strong claims about causality, as neither brain activity nor reports are directly manipulated. We therefore included tests of models with reversed directionality (e.g., cues affect reports, which affects brain activity) in key PPN mediator regions to assess qualitative differences in path coefficients; however, strong inferences about the causality of PPN-pain report relationships are beyond the scope of this paper and are best addressed by invasive manipulation of the brain (Schnitzler and Ploner, 2000; Lefaucheur et al., 2001; GraffGuerrero et al., 2005; Bestmann et al., 2008; Nahmias et al., 2009).

Finally, a mediation test provides inference on whether the inclusion of brain activity $(M)$ in the model explains a significant amount of the covariance between pain predictive cue $(X)$ and reported pain $(Y)$. In the case of single-level mediation, this is equivalent to testing the product of the path coefficients $a \times b$ (Baron and Kenny, 1986; Shrout and Bolger, 2002; Kenny et al., 2003), i.e., c-c' $=a \times b$ (MacKinnon et al., 1995). The mediation test thus assesses whether the mediator explains a significant amount of the effect of the manipulated variable on the measured outcome. It involves tests comparing the strength of the relationships between variables across two separate regression equations rather than testing unique variance associated with two regressors in the same model.

We have developed a multilevel formulation of the path model that provides a voxelwise, mixed-effects mediation analysis that assesses relationships between the task design, brain activity, and behavior and treats the participant as a random effect (see Wager et al., 2009 for details of the method). This analysis can provide information about brain-behavior relationships at two levels. The first level accounts for the relationships between dynamic variations across time (within individual participants) in cue-evoked expectancy, brain activity, and pain reports. Previous work has examined dynamic report-related activity in the context of manipulated expectancies (Koyama et al., 2005). Mediation analysis extends this research by testing whether activity in voxels formally links cue-based expectancy effects to reports. Controlling for cue (path $b$ ) ensures that brain activity actually predicts variations in report, avoiding regions whose activity covaries with reports simply because both are independently affected by cues with no direct brain-behavior relation- ship. The second level tests for consistency across individuals, allowing population inference, and accounts for known sources of variations in individual pathway strength (i.e., person-level moderators) (Kenny et al., 2003). Multilevel mediation analysis differs from standard betweensubjects brain-behavior correlations, because correlations across subjects only capture whether individual differences in brain activity (e.g., average reductions in PPN responses under placebo) and behavior (e.g., average reductions in reported pain) are related. They cannot provide a full account of the mechanisms by which expectancies dynamically modulate behavior (e.g., links between expectancy, brain activity, and trial-by-trial pain reports within subjects). Multilevel mediation analysis provides more direct evidence of the ongoing relationships between painpredictive cue, brain activity, and reported pain within subjects.

Critically, the $a \times b$ mediation test in a multilevel path model is not equivalent to testing whether the conjunction (intersection) of both path $a$ and path $b$ effects are significant. In a single-level path model, the mediation test is significant in a subset of models that show both $a$ and $b$ effects. However, multilevel mediation can identify regions that show evidence for mediation even if the average $a$ and $b$ effects are not significant alone because of an additional component for the covariance (cov) of path $a$ and path $b$ estimates. This is expressed concisely by Kenny et al. (2003) in their Equation 9, which captures the following relationship: mean $(a \times b)=\operatorname{mean}(a) \times$ mean $(b)+\operatorname{cov}(a, b)$. Hence, the $a \times b$ effect can be driven by two different sources: the product of the means of $a$ and $b$ and the covariance between $a$ and $b$. Thus, part of what creates a significant mediation effect is whether path $a$ (cue effects) and path $b$ (report-related responses) show reliable relationships; but another part is whether the individuals who show strong path $a$ effects also show strong path $b$ effects. This allows us to identify voxels that contribute to the overall relationship between cue and pain report but show individual differences in each of these paths (i,e. differences in regression slopes). In the case of positive mediation driven by covariance, subjects who show greater activity in response to high-pain cues also report greater pain with greater activity in the region, while subjects who show less activity in response to high-pain cues report greater pain with less activity. The functional significance of such regions, which contribute strongly to the positive relationship between cues and reported pain in both cases, would never be identified through standard regression analyses of path $a$ or path $b$ alone, which only identify regions with consistent effects across subjects. These patterns may point to the existence of a second-level moderator (i.e., individual differences variable) that might explain the covariance between the paths; here, we test whether individual differences in pretask expectancy help explain variations in within-subjects pathway strength.

We implemented the multilevel path analysis in a voxelwise analysis framework that we have termed "mediation effect parametric mapping” (MEPM) (Wager et al., 2008, 2009) using a custom Matlab toolbox (T.D.W.). MEPM was conducted as by Wager et al. (2008, 2009), with additional facilities incorporated to address the multilevel case. We used bootstrap significance testing, which provides more sensitive tests of mediation (Bollen and Stine, 1990; Stone and Sobel, 1990; Efron and Tibshirani, 1993; Shrout and Bolger, 2002). In our second-level analysis, we employ Efron's bias-corrected, accelerated bootstrap (Efron and Tibshirani, 1993) to test the significance of all effects $(a, b$, and $a \times b)$. We estimated distributions of subject level path coefficients by randomly sampling with replacement $10,000 \mathrm{ob}-$ servations (rows) from the matrix of $[a b a \times b]$ path coefficients for each voxel. Two-tailed, uncorrected $p$ values were calculated from the bootstrap confidence interval.

In sum, the multilevel MEPM framework allowed us to test mediation effects on each voxel in a search region and identify brain voxels that show path $a$, path $b$, and mediation $(a \times b)$ effects. In analysis 1 we identified brain voxels whose heat-evoked responses mediated cue effects on reported pain, focusing on mediators identified within the PPN. In analysis 2 , we searched for voxels whose anticipatory responses contributed to (i.e., mediated) cue effects on the PPN mediators identified in analysis 1. 


\section{Results}

We used MEPM to construct within-subject statistical parametric maps of cue effects, report-related responses, and mediation effects. We focus here on fMRI responses during pain that were identified within pain-processing networks of a priori interest, as defined based on an intensity-processing localizer in an independent sample (see Fig. S1, available at www.jneurosci.org as supplemental material), as well as frontal and limbic regions known to be involved in value processing and cognitive control based on previous literature. Other results are presented in supplemental figures and tables for completeness. The threshold used for all analyses was $p<0.001$ with a cluster extent of at least three contiguous voxels, a consensus threshold (Wager et al., 2007a) that also provided familywise error rate corrected results at $p<$ 0.05 within each of our a priori PPN regions of interest (tested using the AFNI AlphaSim program) (Cox, 1996). We visualized all results in MNI space overlaid on the mean T1 image derived from the genetic algorithm normalization and verified localization using the SPM Anatomy toolbox version 1.5 (Eickhoff et al., 2007).

\section{Conscious expectancies}

Subjects expected high-pain stimulation in response to the highpain cue $(M=7.45, \mathrm{SD}=0.47)$ and low-pain stimulation in response to the low-pain cue $(M=2.04, \mathrm{SD}=0.77)$, both before any cue-heat pairings were experienced (before reinforcement) and throughout the task. The magnitude of the difference between cue-related expectancies (high-low) was stable across the entire experiment (effect of time: $t_{(18)}=0.75, p>0.1$ ), suggesting that expectancies were strong and consistent throughout the experiment. Thus, participants did not behave as though they were aware that some trials were medium temperature trials in the later runs. To identify the extent to which conscious expectations played a role in cue effects on pain responses, we tested whether cue effects and mediation effects correlated with individual differences in pretask expectancy. We report these results in the following sections.

\section{Reported pain}

First, we examined the effect of pain predictive cue (high vs low) and noxious stimulus level (high, medium, low) on reported pain. Participants reported greater pain with increasing temperature $\left(t_{(17)}=8.20, p<0.0001\right)$. The critical mediumtemperature trials were rated as substantially more painful when preceded by high-pain cues than when preceded by low-pain cues $\left([\mathrm{HM}-\mathrm{LM}] ; t_{(17)}=8.59, p<0.0001\right)$; on the 8-point rating scale, this corresponded to an average difference of 1.6 , or $\sim 20 \%$ (Fig. 1). The magnitude of this effect remained consistent throughout the experiment; there was no evidence for any effect of time on perceived pain $\left(t_{(17)}=0.046, p=0.65\right)$, nor was there any interaction between time and cue effects on pain $\left(t_{(17)}=1.11\right.$, $p=0.28$ ) (see Figure S2, available at www.jneurosci.org as supplemental material). In the mediation framework, this establishes the existence of a relationship between predictive cues and reported pain (Fig. 2, path $c$ ). We searched for brain mediators of this relationship (analysis 1 ).

\section{Intensity processing}

To verify that PPN intensity-related processing was intact in the presence of manipulated expectancies, we compared responses on validly cued high- and low-intensity trials [HH - LL]. Consistent with extant literature on pain processing in the central nervous system, we observed pain-related activity $(\mathrm{HH}>\mathrm{LL})$ throughout the PPN, as defined by our independent localizer. Specifically, we observed intensity-related differences in right secondary somatosensory cortex (SII), right thalamus, right dorsal anterior cingulate cortex (dACC), right mid-cingulate, bilateral anterior and mid-insula, bilateral and medial cerebellum, and a region of the midbrain that includes the periaqueductal gray (PAG) (Figs. S1, S3; the full set of voxelwise results is presented in Table S1, available at www.jneurosci.org as supplemental material). We also tested whether intensity affected pain-evoked responses in the PPN, controlling for the effect of cue ( $\mathrm{HH}-\mathrm{HM})$. This analysis revealed intensityrelated differences $(\mathrm{HH}>\mathrm{HM})$ in right $\mathrm{SII}$, dACC, middle cingulate, and right anterior and mid-insula (Fig. S3, Table S2, available at www.jneurosci.org as supplemental material). These intensity-related changes were less robust than in our $\mathrm{HH}$ - LL contrast, as expected given the smaller temperature difference and thus reduced power. Nonetheless this contrast indicates that key PPN regions respond to changes in stimulus intensity when cue is held constant.

\section{Cue-based modulation of pain activity (analysis 1, path a)}

The critical [HM - LM] comparison identified brain regions in which activity during a single level of noxious stimulation varies as a function of pain-predictive cue (cue effects, path $a$ ) (Fig. 2). This contrast revealed a network of regions that showed greater activity during thermal stimulation when preceded by high-pain cues rather than low-pain cues. These regions are shown in yellow in Figure 3 and supplemental Figure S4 (available at www. jneurosci.org as supplemental material). Importantly, all PPN regions identified via our independent localizer and our intensity-processing contrast within this sample were more active following high-pain cues, except PAG and dACC; specifically, cuebased modulation was observed in right SII/dorsal posterior insula, right thalamus, medial thalamus, left anterior insula, bilateral cerebellum, and right pre-supplementary motor area (SMA) (Table S3, available at www.jneurosci.org as supplemental material).

These regions can be divided into those that peaked early in noxious stimulation (left anterior insula, medial thalamus, right cerebellum, and right pre-SMA) and those that peaked later in stimulation (right SII/insula, right thalamus, and left cerebellum). These regions generally displayed cue effects that emerged when the region showed its peak response (see Figs. S5, S6, available at www.jneurosci.org as supplemental material). Left anterior insula was the only PPN region that showed cue effects during anticipation, before noxious stimulation $(p<0.001)$, and voxels that showed anticipatory differences did not overlap with those that showed cue effects during stimulation.

Additional regions that showed increased activity following high-pain cues included prefrontal [left dorsolateral prefrontal cortex (DLPFC)], limbic (left amygdala, medial striatum/hypothalamus), and brainstem (pons) regions, among others (Fig. 3; Table S3, available at www.jneurosci.org as supplemental material). Other regions were more active following low-pain cues (LM > HM, shown in blue in Fig. 3 and Fig. S4, available at www.jneurosci.org as supplemental material), including bilateral ventrolateral prefrontal cortex (VLPFC), medial orbitofrontal cortex (OFC), inferior parietal lobule, and sensorimotor cortex (Table S3, available at www.jneurosci.org as supplemental material). For the most part, these effects were driven by greater painevoked deactivation on HM trials, which is consistent with the fact that these regions showed pain-related deactivations in our 


\section{Path a PAIN PREDICTIVE CUE}

a
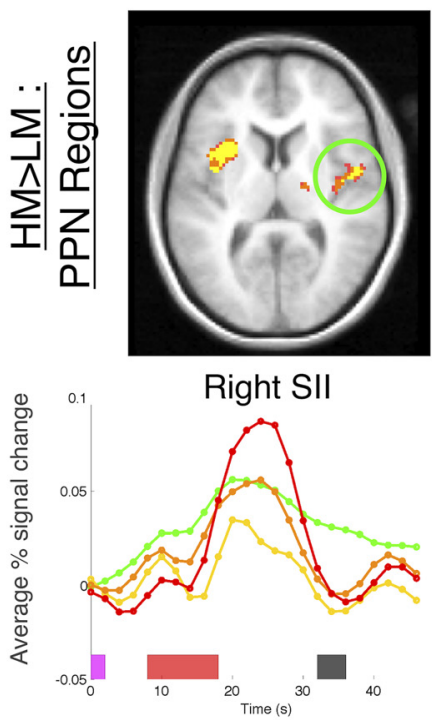

b

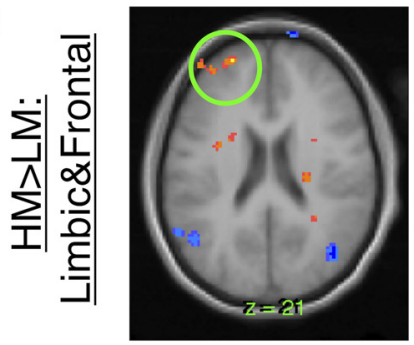

Left DLPFC

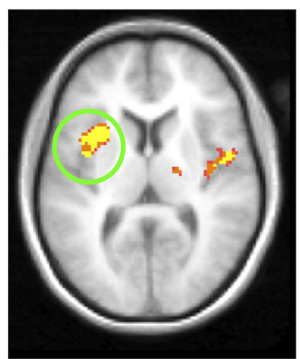

01 Left Anterior Insula
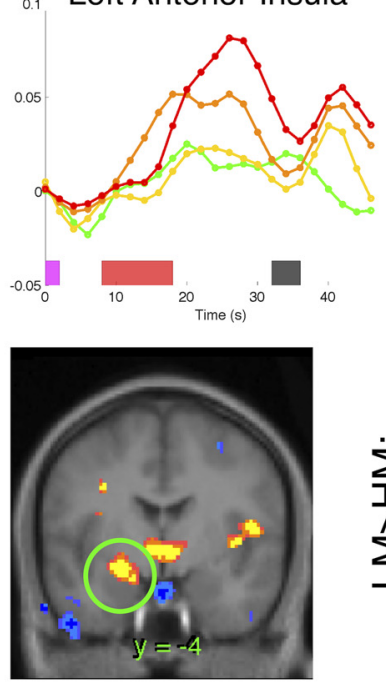

Left Amygdala

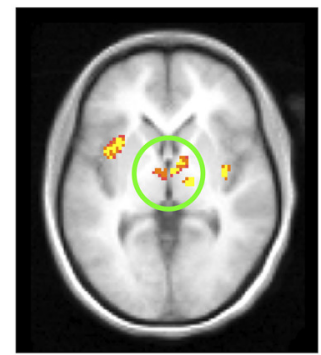

Medial Thalamus
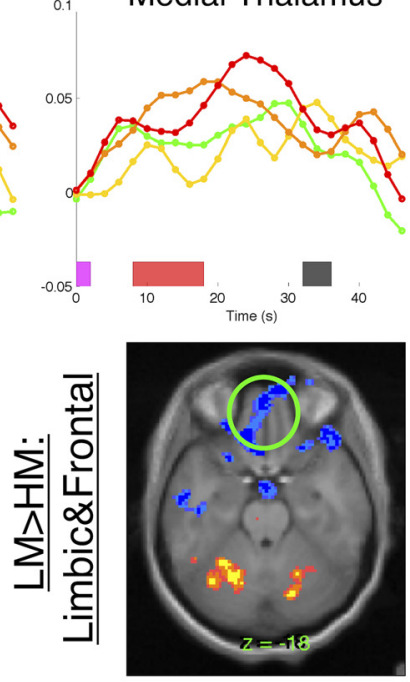

Medial OFC

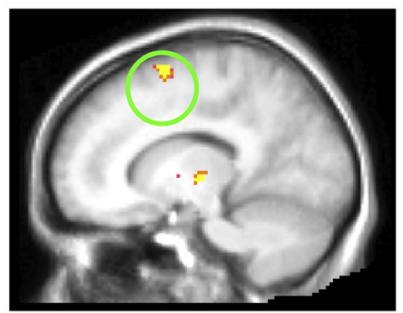

0.15. Right Pre-SMA
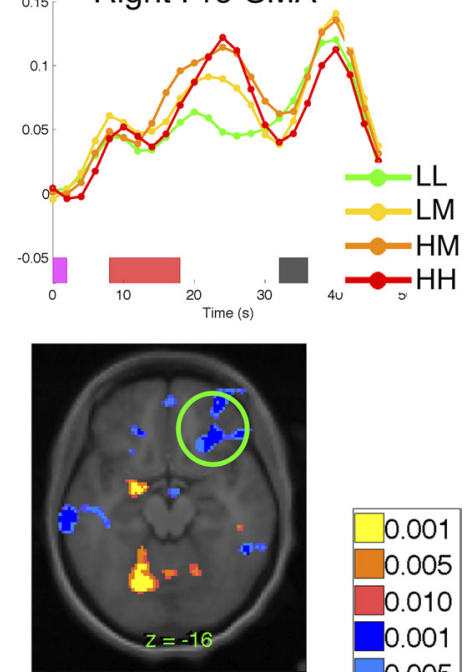

Right VLPFC

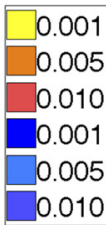

Figure 3. Path $a$ : cue-based modulation of pain period activity. $\boldsymbol{a}$, Cue effects on PPN regions. Our analysis of cue effects ("path $a$ ") consists of a direct contrast between activity on HM and LM trials. Nearly all PPN regions were modulated by predictive cues. Extracted time courses (bottom) demonstrate that responses peak late in the pain period or after termination of the stimulus for most regions of interest, due at least in part to hemodynamic response lag. Pink bars on the $x$-axis indicate cue presentation, red bars indicate noxious stimulation, and gray bars indicate rating scale presentation. $\boldsymbol{b}$, Cue effects on limbic and frontal regions. We also observed positive cue effects (HM $>$ LM, yellow/orange) in left DLPFC and left amygdala, while medial OFC and right VLPFC showed greater activity with low-pain cues (LM $>$ HM, blue).

$\mathrm{HH}$ versus LL intensity-processing contrast (Table S1, available at www.jneurosci.org as supplemental material).

To identify the extent to which conscious expectancies play a role in cue effects on pain responses, we tested whether cue effects correlated with individual differences in expectancy. There was a significant relationship between expectancy and cue effects on left middle insula and right medial OFC $(p<0.001)$, such that those individuals who expected larger differences in noxious stimulation as a function of cue exhibited greater cue effects on responses in these regions (e.g., larger insula increases and medial OFC decreases on HM trials, relative to LM trials).

Activity related to reported pain during medium-heat trials (analysis 1, path b)

We next identified voxels whose activity predicted reported pain on a trial-by-trial basis during HM and LM trials, controlling for cue effects (path $b$ or "report-related responses") (Fig. 2). Regions identified in this analysis predict trial-to-trial variations in reported pain despite the fact that both noxious stimulation and cue were held constant for all trials included in this analysis. Several PPN regions were positively related to reported pain, including right anterior insula, rostrodorsal anterior cingulate (rdACC), left cerebellum, and right pre-SMA (Fig. 4). Additional regions outside the PPN also predicted pain reports, including right dorsomedial prefrontal cortex (dmPFC), right DLPFC, right VLPFC, left thalamus, and right putamen. Regions inversely related to reported pain included bilateral medial OFC, bilateral temporoparietal junction (TPJ), left hippocampus, and right posterior cingulate (Fig. S7, Table S4, available at www.jneurosci.org as supplemental material).

Brain mediators of cue-based expectancy effects on perceived pain (analysis 1, mediation)

Analysis 1 was designed to test whether regions involved in pain processing underlie predictive cue effects on pain and to identify additional networks that contribute to this relationship. We used whole-brain multilevel mediation to identify regions that mediated the behavioral relationship between pain predictive cue (HM vs LM) and perceived pain (trial-by-trial pain reports) (path $a \times b$ ) (Fig. 2). As hypothesized, a subset of PPN regions were identified as positive mediators, including right thalamus, left rdACC, and left anterior insula (Fig. 5). All of these regions showed positive path coefficients for both cue effects and reportrelated responses, suggesting that for most participants these regions showed increases with high-pain cues (HM $>$ LM), and activity increases predicted increased reported pain. We note that while the brain-pain report relationship in this model is correlational and the causal directionality of the brain-report pathway 


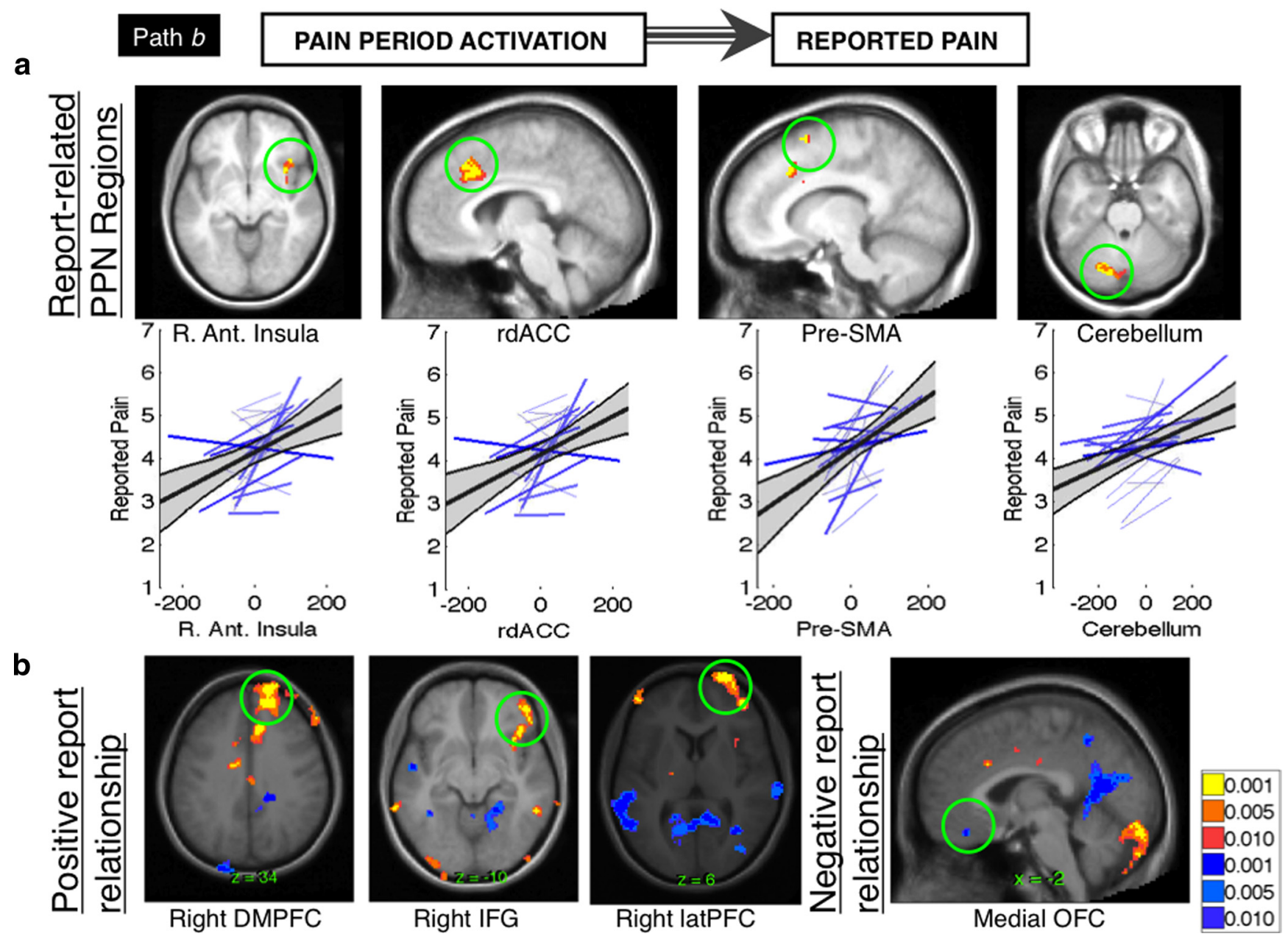

Figure 4. Path $b$ : activity related to reported pain. $\boldsymbol{a}$, Report-related PPN regions. Our pain report analysis ("path $b$ ") identifies voxels whose pain period activity predicts trial-by-trial pain ratings, controlling for cue. This analysis revealed that a subset of pain processing regions, including right anterior insula, rdACC, pre-SMA, and bilateral cerebellum, correlated with variations in subjective intensity under a constant level of noxious thermal stimulation. For each PPN region, bottom graphs show the variability in path $b$ coefficients across individual subjects (blue lines) and the group regression slope (dark black line) and 95\% confidence interval (gray shading). $\boldsymbol{b}$, Report-related frontal and limbic regions. In addition to PPN regions, right dmPFC, inferior frontal gyrus (IFG), and lateral PFC were also positively correlated with trial-by-trial pain reports, controlling for cue (yellow/orange). Medial OFC was inversely related to perceived pain (blue).

cannot be reliably inferred, this forward model fit the data in these regions better than a path model in which reported pain and brain response were reversed (reports lead to pain-evoked responses). The reverse model did not explain activity in left anterior insula or right thalamus (all $p>0.1$ ), and while rdACC was adequately explained by this model $(p<0.05)$, our forward model provided a better fit for activity in this region $(p<0.005)$.

Interestingly, only left anterior insula showed consistent effects at the group level in all three paths. This suggests that for the two remaining regions there was substantial variability across individuals within individual paths. Because these regions were both mediators of the predictive cue-pain report relationship and located in the PPN as defined by an independent localizer, we refer to these regions as "pain processing network mediators" (PPNMs).

A second set of regions outside the PPN showed significant positive mediation effects as well, including pons, left putamen, left caudate, left dorsal amygdala, and left DLPFC (Fig. S8, Table S5, available at www.jneurosci.org as supplemental material). These regions have been associated with expectancy and affective value across many studies (Kober et al., 2008). Thus, they might influence pain reports independently of the PPN, suggesting purely affective or decision-based influences on pain reporting, or they might have indirect effects on pain via interactions with PPNMs.

Other regions were identified as positive mediators because they showed negative expectancy effects (greater pain-period activity with low-pain expectancy; LM $>$ HM) and because relative deactivation predicted increased pain (negative report-related responses). This profile was most notably observed in pregenual anterior cingulate cortex (pgACC), a region that shows increased opioid release under placebo (Wager et al., 2007b) and has been thought to modulate relevant subcortical processes in pain (Petrovic et al., 2002; Bingel et al., 2006; Eippert et al., 2009) and emotion (Petrovic et al., 2005). Additional regions that exhibited similar patterns are shown in Figure S8 and Table S5, available at www.jneurosci.org as supplemental material.

We also tested whether the strength of the mediation effects $(a \times b$ product $)$ correlated with individual differences in pretask expectancies. We found that the strength of the mediation effect in left DLPFC and right VLPFC was predicted by expectancy scores, suggesting that mediation in these regions is likely to be related to conscious expectations. 
a

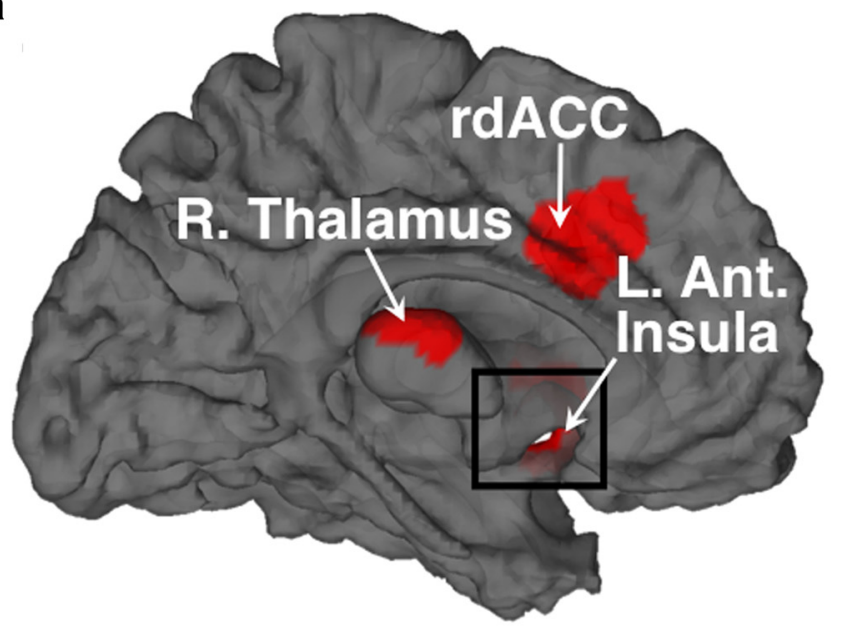

b
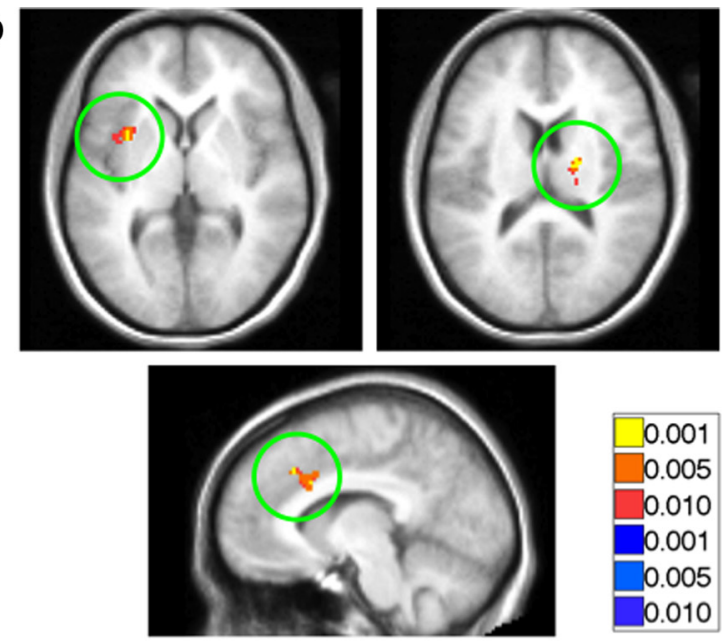

0.001

0.005

0.010

0.001

0.005

0.010

C

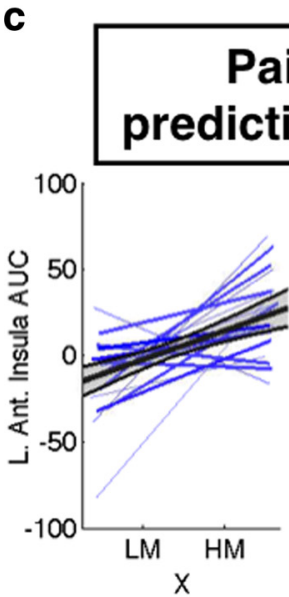

Direct effect (c')

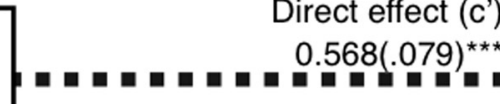
cain cue

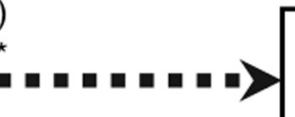

\section{Reported Pain}

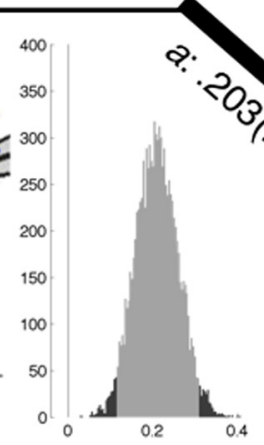

L. Ant. Insula Mediation Effect
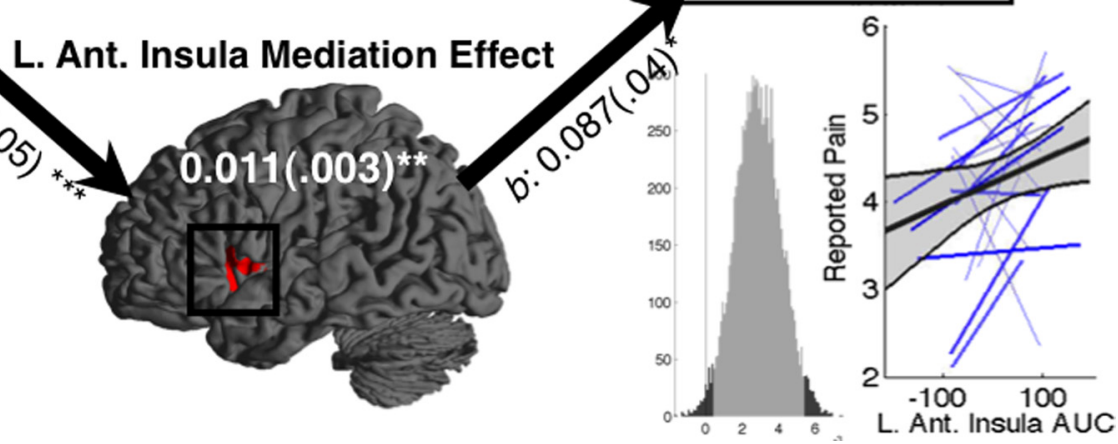

Figure 5. Pain processing network mediators. $\boldsymbol{a}$, Pain processing network mediators: surface map. Three key PPN regions were found to mediate expectancy effects on perceived pain. $R$. Thalamus, Right thalamus; L. Ant. Insula, left anterior insula. $\boldsymbol{b}$, Pain processing network mediators. Slice views of PPNMs, clockwise from top left: left anterior insula ( $-36,6,-4)$; right thalamus $(18,-18,14)$; left rdACC $(-8,18,32)$.c, Mediation path diagram for the left anterior insula. Left anterior insula shows a positive path a effect, indicating more activity with high-pain cues. The mean standardized path coefficient is shown with standard error (in parentheses). Lower left, Individual subjects' regression lines and group average, as in Fig. 4. Lower right, Histogram of the bootstrap estimates of the path a distribution. The light shading shows the $95 \%$ bootstrap confidence intervals, and vertical line marks the null hypothesis value of zero. The right panels show the path $b$ effect, which shows a link with trial-by-trial reported pain. Standardized path coefficients and standard error for the mediation effect are shown in white text on the brain surface map. The dashed arrow represents the direct effect of predictive cues on perceived pain (path $\left.c^{\prime}\right) .{ }^{*} p<0.05,{ }^{* *} p<0.01,{ }^{* * *} p<0.001$.

Functional response profiles in key mediator regions

Our mediation analyses indicate that responses evoked by the critical medium temperature trials in the PPN and other regions track expected intensity and subtle variations in reported pain. This would be consistent with a signal that assimilates nociceptive processes with previous expectations and thus shows monotonic effects of both stimulus intensity and pain-predictive cue (e.g., $\mathrm{LL}<\mathrm{LM}<\mathrm{HM}<\mathrm{HH}$ ). However, positive cue effects (HM $>$ LM) and concomitant changes in report can be created by other kinds of responses, such as prediction error signaling or tracking expected value, which would imply different computational processes. As shown in Figure 6, we examined the pattern of responses in each mediator region to compare the assimilation account with several alternatives. One alternative is that regions may be driven only by expectations. These regions would show a cue-based effect on medium trials (HM > LM), but pain period responses would be driven entirely by the cue's expected value, resulting in a step function (e.g., [LL and $\mathrm{LM}]<[\mathrm{HM}$ and $\mathrm{HH}]$ ). Another alternative is that cue effects emerge in regions that are sensitive to expectancy violations. These expectancy violations may be coded as either valence-specific (signed) or salience (un- signed, absolute value) prediction errors (O'Doherty et al., 2003; Jensen et al., 2007). A region supporting a salience signal or unsigned prediction error would show greater responses to expectancy violations (medium trials) than trials in which stimulus intensity was validly cued (e.g., $[\mathrm{HM}>\mathrm{LM}]>[\mathrm{LL}$ and $\mathrm{HH}]$ ). Alternatively, expectancy effects may emerge in regions that show patterns consistent with aversive or appetitive prediction errors. In this task, LM trials were more painful than expected, which would presumably result in an aversive prediction error. HM trials were less painful than expected, which would elicit an appetitive prediction error.

As shown in Figure 6, responses in pons, right thalamus, left anterior insula, and left DLPFC (Fig. 6, red) showed the predicted assimilation pattern, suggesting that their responses are a combination of nociceptive signals and previous cue-related expectations. PgACC showed a similar pattern, with opposite sign, which is consistent with its role in pain modulation. However, other regions showed different patterns. Responses in the dACC (Fig. 6, orange) were best described as a pure expectancy effect, as there was a large expectancy effect and little evidence for differences between low and medium temperature with low expectancy and 

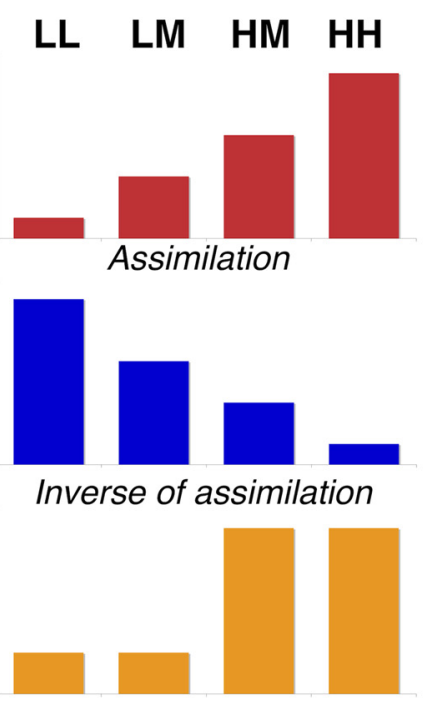

\section{Expected Intensity}

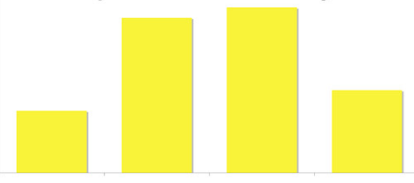

Expectancy violation
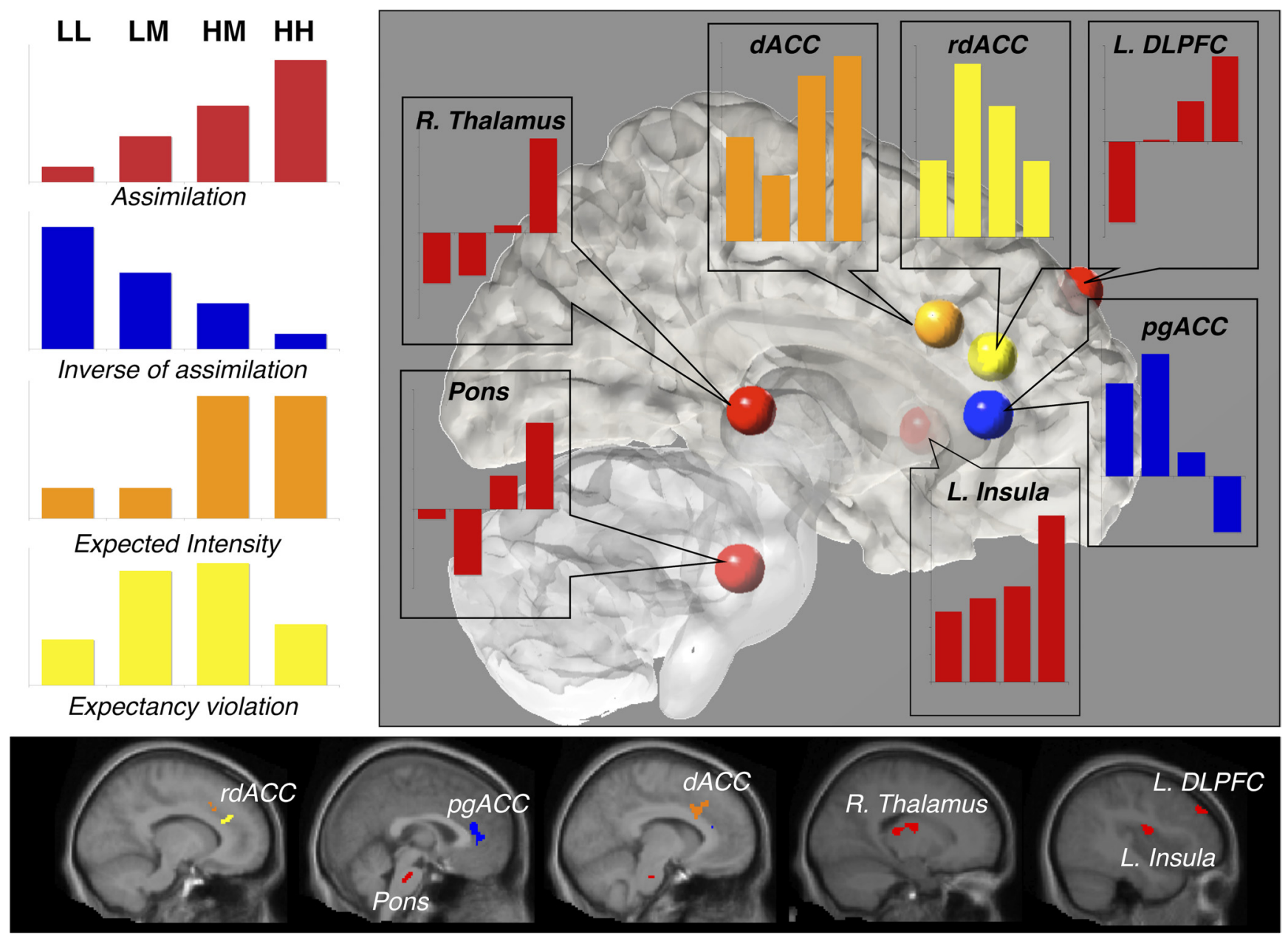

Figure 6. Functional response profiles in key mediator regions. Bar graphs depict functional patterns underlying observed cue effects in key mediator regions. The left panel illustrates theoretical patterns across levels of noxious stimulation. Regions depicted in red, including left anterior insula (L. Insula), right thalamus (R. Thalamus), left dorsolateral prefrontal cortex (L. DLPFC), and pons display a pain assimilation profile ( $\mathrm{LL}<\mathrm{LM}<\mathrm{HM}<\mathrm{HH}$ ). Conversely, pregenual anterior cingulate, depicted in blue, shows a response profile that is inversely related to the assimilation pattern $(\mathrm{LL}>\mathrm{LM}>\mathrm{HM}>\mathrm{HH}$ ). Dorsal anterior cingulate cortex, depicted in orange, shows a profile based on expected intensity, such that responses to the high-pain cue conditions (HH and HM) are greater than responses to the low-pain cue conditions (LL and LM). Rostrodorsal anterior cingulate, depicted in yellow, shows an expectancy violation signal, such that responses to medium trials are greater than responses to either of the validly cued conditions.

between medium and high temperature with high expectancy. A neighboring region, rdACC (Fig. 6, yellow), showed evidence for a salience effect: responses were highest in the medium-temperature conditions when expectancies were violated.

Relationships between cue-evoked anticipatory responses and pain processing network activity (analysis 2 )

The mediation analyses above identified both PPNMs (Fig. 5) and other key regions of theoretical interest that are not typically associated with pain. A key question concerns the brain mechanisms that lead to cue-related differences in PPNMs. If expectancy effects result from a change in the motivational significance of pain (Fields, 2007), it is likely that effects on PPNMs are mediated by changes in cognitive systems thought to maintain expectations in working memory (e.g., DLPFC) and systems involved in learning and affective value (e.g., medial OFC, amygdala, and striatum).

In analysis 2, we asked whether cue-evoked anticipatory responses predicted the magnitude of PPNM pain-evoked responses on medium-temperature trials. In this case, a significant mediation effect entails a relationship between pain-predictive cue and cue-evoked anticipatory activity in the region and between the region's anticipatory activity and average PPNM activity during noxious stimulation (Fig. 7). We conducted wholebrain multilevel mediation to identify mediators of average PPNM activity and then tested the relationships between mediators and each of the three PPNMs (insula, thalamus, and rdACC). To constrain our interpretations, we report only the regions that positively mediated pain period responses in all three individual PPNM regions. This analysis revealed that cue effects on PPNM noxious stimulus-evoked activity were positively mediated by anticipatory cue-evoked responses in medial OFC and right ventral striatum (Fig. 7, Table 1).

\section{Negative mediators (Mediation, path $\boldsymbol{a} \times \boldsymbol{b}$ )}

In this report our a priori hypotheses focused on positive mediators, the brain regions that contribute to the robust relationship between expectancy and reported pain. However, mediation analysis can unpack multiple effects that contribute to observed outcomes. In addition to the positive mediators identified above, it is likely that additional processes such as preparation (Weisenberg et al., 1996) and conditioned analgesic responses (McNally, 1999), might act to decrease pain under high-pain expectancy. 
These effects would be masked by the robust pronociceptive expectancy effects in this paradigm (referred to as "suppression" in the mediation literature) (MacKinnon et al., 2000), but they may still be detectable as patterns of fMRI responses. Specifically, the brain mechanisms underlying anti-nociceptive effects of expectancy would appear as negative mediators in our analysis. Negative mediators that showed positive predictive cue effects (increases with high-pain cues) and negative report-related responses (anti-nociceptive effects) included left SII, right caudate nucleus, left internal globus pallidus, and others (Table S6, Fig. S8, available at www. jneurosci.org as supplemental material). Those with negative predictive cue effects (more activation when less pain was expected) and positive report-related responses included middle cingulate cortex, right sgACC/OFC, and several regions in prefrontal cortex (right dmPFC, right DLPFC, right lateral PFC, left inferior frontal gyrus), among others. The presence of negative mediators suggests that there may be multiple processes activated by expectancy cues, some of which exacerbate pain and others that ameliorate it. These results were not part of our a priori hypotheses but could lead to additional studies to unpack the complexity inherent in that expectations for high pain can both enhance pain and lead to analgesic preparatory effects (Fanselow, 1986).

\section{Discussion}

Informational cues, which induce expectancies (Kirsch, 1985; Rescorla, 1988), have widely appreciated effects on visual perception (Kastner et al., 1999), but there is less data on how they shape affective processes such as pain. In this study, we use a new analysis strategy to identify brain mediators of predictive cue effects on perceived pain. We isolate the subset of pain-processing network regions that are most directly responsible for constructing experienced pain based on cue-related expectations and show that changes in these regions are predicted by anticipatory responses in medial OFC and ventral striatum.

\section{PPN regions mediate cue effects on behavior}

Expectations about stimuli and/or treatments have been shown to affect brain responses in the so-called "pain matrix" or PPN (Petrovic et al., 2002; Wager et al., 2004; Koyama et al., 2005; Price et al., 2007), although not all studies find such effects (Keltner et al., 2006; Kong et al., 2006). However, demonstrating PPN modulation does not provide strong evidence that these effects drive subjective experience. There are many examples of stimulus effects on brain activity that do not appear to contribute to conscious perception, such as amygdala responses to unperceived laser stimulation (Bornhövd et al., 2002) or masked fearful faces (Whalen et al., 1998). Mediation analysis formally tests whether a functional pathway links cues, brain activity, and experienced pain.

We found robust effects of predictive cues on the PPN (path $a$ ) as identified using an independent localizer, with greater activity seen with high-pain cues in all PPN regions except dACC (but see below) and PAG. Pretask expectancies correlated with cue-based modulation in left insula and medial OFC, providing direct evidence that expectations influence pain-evoked responses.

Several PPN regions, including rdACC, anterior insula, preSMA, and bilateral cerebellum, predicted trial-by-trial variations in pain reports at a constant temperature and controlling for manipulated cues (path $b$ ). Previous studies have shown increases in activity in similar regions with parametric increases across stimulus intensities (Coghill et al., 1999; Craig et al., 2000; Bornhövd et al., 2002; Helmchen et al., 2008). Our results could reflect endogenous variation in pain processing due to fluctuations in arousal, attention, or related factors, and they build on recent work relating pain to ongoing fluctuations in brain activity (Boly et al., 2007; Ploner et al., 2010).

Most importantly, three PPN regions (rdACC, anterior insula, and thalamus) mediated cue effects on reported pain. These regions showed increased pain-evoked responses following highpain cues and, controlling for cue, greater activity predicted higher pain. Notably, other PPN regions that responded strongly to cues did not show evidence for mediation, demonstrating a distinction between regions that directly integrate previous information and nociception (PPNMs), those that are modulated by cues but relate only indirectly to perception (e.g., SII), and those unaffected by cues (e.g., PAG).

\section{Cue-based modulation versus placebo analgesia}

Placebo analgesia decreases pain-evoked responses in anterior insula, dACC, and thalamus (Wager et al., 2004; Price et al., 2007; Eippert et al., 2009), and individual differences in reported placebo analgesia are correlated with effects on these regions (Wager et al., 2004). Our results conceptually replicate these findings and 
Table 1. Analysis 2: relationships between cue-evoked anticipatory responses and pain-processing network mediator activity

\begin{tabular}{|c|c|c|c|c|c|c|c|c|c|c|}
\hline \multirow[b]{2}{*}{ Brain region } & \multicolumn{3}{|c|}{ Coordinates (mm) } & \multirow{2}{*}{$\begin{array}{l}\text { No. of voxels } \\
(p<0.001)\end{array}$} & \multirow[b]{2}{*}{ Volume (mm) } & \multirow[b]{2}{*}{ PPNM $a \times b$ effect } & \multirow[b]{2}{*}{$\operatorname{PPNM} \operatorname{cov}(a, b)$} & \multirow[b]{2}{*}{ Insula $a \times b$ effect } & \multirow[b]{2}{*}{ Thalamus $a \times b$ effect } & \multirow[b]{2}{*}{$\operatorname{rdACC} a \times b$ effect } \\
\hline & $x$ & $y$ & $z$ & & & & & & & \\
\hline Medial OFC & -4 & 60 & -14 & $0.7952(0.18)^{* *}$ & 1016 & $0.7952(0.18)^{* *}$ & 1.3977 & $0.8566(0.51)^{\perp}$ & $0.748(0.25)^{* *}$ & $0.9488(0.32)^{* *}$ \\
\hline Right ventral striatum & -10 & 4 & -14 & $0.3345(0.09)^{* *}$ & 808 & $0.3345(0.09)^{* *}$ & 0.8608 & $0.5048(0.004)^{* *}$ & $0.3092(0.16)^{*}$ & $0.4471(0.03)^{*}$ \\
\hline
\end{tabular}

This table presents results from analysis 2, presented as a path diagram in Figure 7. Activity was averaged across pain processing network mediators (rdACC, left anterior insula, and right thalamus), and we searched for regions whose cue-evoked responses during medium trials mediated cue effects on average PPNM responses during pain. We then tested the relationship between each anticipatory mediator and the three PPNM regions. This table presents results for medial $0 \mathrm{FC}$ and right ventral striatum, which were the only regions whose anticipatory cue-evoked responses positively mediated cue effects on pain-evoked responses in all three PPNMs. ${ }^{*} p<0.05,{ }^{* *} p<0.01,{ }^{\perp} p=0.0818$.

show that they can be produced by short-term predictive cues as well: Each of these PPN regions mediated cue effects on trial-totrial pain reports.

Our results also suggest that placebo analgesia and cuebased effects involve modulation by common regions outside the PPN. Placebo analgesia studies point to modulatory roles of rostral ACC/pgACC, DLPFC, OFC, and VLPFC (Petrovic et al., 2002; Lieberman et al., 2004; Wager et al., 2004; Bingel et al., 2006). In this study, each of these regions mediated cue effects on reported pain. Pretask expectancies correlated with effects in left DLPFC and right VLPFC, suggesting that mediation by these regions may relate to conscious expectations. In addition, pgACC showed negative cue effects (increased activity with low-pain cues) and negative report-related responses (greater activity led to lower pain). This is consistent with studies of pain and emotion, which suggest that placeboinduced increases in pgACC lead to context-based modulation (Petrovic et al., 2002, 2005; Bingel et al., 2006; Eippert et al., 2009). This shared functional significance would not have been apparent without linking cue effects on this region to pain reports using mediation.

There are also important differences between placebo analgesia and cue-based pain modulation. Placebo analgesia is likely to involve tonic, sustained processes such as opioid and dopamine release and affective shifts (Benedetti et al., 1999; Zubieta et al., 2005; Fields, 2007; Scott et al., 2007; Wager et al., 2007b; Craggs et al., 2008). Sustained neuromodulatory processes are not a viable explanation when cues vary on each trial. This may explain why we did not observe cue effects on the PAG, a PPN region critical for endogenous opioid-based analgesia (Fields, 2004) that is modulated by placebo (Wager et al., 2004; Eippert et al., 2009). Instead, cue effects must depend on more transient processes. A wealth of cross-species research on learning and evaluation suggests that the OFC works together with the striatum and amygdala to evaluate predictive cues and generate value and prediction error signals (Gottfried et al., 2003; Saddoris et al., 2005; Schoenbaum et al., 2007). These processes, discussed below, are likely to differentiate cue-based effects from placebo effects on brain activity.

\section{Anticipatory mediators}

Cue-evoked responses in medial OFC and right ventral striatum mediated cue effects on PPNM pain-evoked responses. On average, these regions showed greater activity with highpain cues, and greater anticipatory activity led to higher PPNM activity during medium heat. According to current theories, these two regions support value and prediction error calculation and thereby underlie the development of cuebased predictions and trial-to-trial changes in appetitive and aversive value. Medial OFC/VMPFC is linked to the generation of positive and negative value (Tom et al., 2007; Hare et al., 2008), and ventral striatum shows increased activation in anticipation of both monetary reward (Knutson et al., 2001) and aversive shock (Jensen et al., 2003). Our findings are also consistent with work on dynamic temporal difference models of reinforcement learning in the context of aversive stimulation (Seymour et al., 2005; Menon et al., 2007; Delgado et al., 2008). Thus, circuitry involved in generating value signals is also important for shaping PPNM pain-evoked responses, which are in turn linked to pain perception.

\section{Future directions}

This report focused on whether PPN regions mediate cue effects on pain. Our analyses also identified mediators and report-related regions outside the PPN. Although pain reports are the best currently available measure of pain, they rely on subjective decisions and may be affected by decision biases independently of nociceptive processes (Yang et al., 1985). A direct analysis of the relationship between non-PPN report-related regions and PPNMs could identify the extent to which reports rely on sensory changes versus biased decision making.

To maximize cue effects on reported pain, we used both instructions and reinforcement throughout the experiment. While we have direct evidence that pretask expectancies correlated with cue effects on pain-evoked responses, cognitively impenetrable associative learning may also play a role (Benedetti et al., 2003). It was not possible to differentiate conscious and nonconscious processes in the current experiment, since we examined responses after learning reached asymptote. Disentangling these processes is an important goal for future work.

Future studies should address several limitations of our experimental design. Sustained noxious thermal stimulation may be more sensitive to expectancies than transient modalities such as laser stimulation; future work should test whether effects differ as a function of stimulus modality. Our rating scale was anchored in terms of pain tolerance, which can be dissociated from judgments of pain magnitude; although our comparisons were mainly within participants, this may affect individual differences analyses (Chapman et al., 1985; Robinson et al., 2003). Previous work found that expectations for decreased pain, but not increased pain, modulated perception (Koyama et al., 2005). This is consistent with our findings, as LM ratings were substantially lower than the medium stimulus's calibrated VAS score of 5, whereas HM ratings were not significantly different from calibrated levels (see Fig. 1). Future work should incorporate a validly-cued medium condition to directly test whether [HM - LM] effects reflect pain enhancement by high-pain cues, pain reduction by low-pain cues, or equal contributions of the two.

Finally, mediation does not imply causality unless both input (cue) and mediator (brain) are manipulated (Holland, 1988; Sobel, 2008). While we can infer that cues causally affect PPN responses, we need converging evidence from experimental manipulations of brain activity (i.e., with brain stimulation) to com- 
pletely demonstrate that PPNM activity is causally linked to behavioral outcomes.

\section{Summary}

Pain-predictive cues and the expectations they engender strongly influence core pain-processing circuits. Anterior cingulate cortex, anterior insula, and thalamus formally mediate cue effects on reported pain and are therefore likely to be critical for the modulation of pain by expectation. Cue effects on these regions are, in turn, mediated by anticipatory responses in medial OFC and ventral striatum, suggesting that these regions are involved in generating value signals that influence pain processing and thus contribute to context-based pain modulation.

\section{References}

Apkarian AV, Bushnell MC, Treede RD, Zubieta JK (2005) Human brain mechanisms of pain perception and regulation in health and disease. Eur J Pain 9:463-484.

Ashburner J, Friston KJ (2005) Unified segmentation. Neuroimage 26:839-851.

Baron RM, Kenny DA (1986) The moderator-mediator variable distinction in social psychological research: conceptual, strategic, and statistical considerations. J Pers Soc Psychol 51:1173-1182.

Benedetti F, Arduino C, Amanzio M (1999) Somatotopic activation of opioid systems by target-directed expectations of analgesia. J Neurosci 19:3639-3648.

Benedetti F, Pollo A, Lopiano L, Lanotte M, Vighetti S, Rainero I (2003) Conscious expectation and unconscious conditioning in analgesic, motor, and hormonal placebo/nocebo responses. J Neurosci 23:4315-4323.

Bestmann S, Ruff C, Blankenburg F, Weiskopf N, Driver J, Rothwell J (2008) Mapping causal interregional influences with concurrent TMS-fMRI. Exp Brain Res 191:383-402.

Bingel U, Tracey I (2008) Imaging CNS modulation of pain in humans. Physiology 23:371-380.

Bingel U, Lorenz J, Schoell E, Weiller C, Büchel C (2006) Mechanisms of placebo analgesia: rACC recruitment of a subcortical antinociceptive network. Pain 120:8-15.

Bingel U, Schoell E, Büchel C (2007) Imaging pain modulation in health and disease. Curr Opin Neurol 20:424-431.

Bollen KA, Stine R (1990) Direct and indirect effects: classical and bootstrap estimates of variability. Sociol Methodol 20:115-140.

Boly M, Balteau E, Schnakers C, Degueldre C, Moonen G, Luxen A, Phillips C, Peigneux P, Maquet P, Laureys S (2007) Baseline brain activity fluctuations predict somatosensory perception in humans. Proc Natl Acad Sci U S A 104:12187-12192.

Bornhövd K, Quante M, Glauche V, Bromm B, Weiller C, Büchel C (2002) Painful stimuli evoke different stimulus-response functions in the amygdala, prefrontal, insula and somatosensory cortex: a single-trial fMRI study. Brain 125:1326-1336.

Büchel C, Bornhövd K, Quante M, Glauche V, Bromm B, Weiller C (2002) Dissociable neural responses related to pain intensity, stimulus intensity, and stimulus awareness within the anterior cingulate cortex: a parametric single-trial laser functional magnetic resonance imaging study. J Neurosci 22:970-976.

Chapman CR, Casey KL, Dubner R, Foley KM, Gracely RH, Reading AE (1985) Pain measurement: an overview. Pain 22:1-31.

Chib VS, Rangel A, Shimojo S, O’Doherty JP (2009) Evidence for a common representation of decision values for dissimilar goods in human ventromedial prefrontal cortex. J Neurosci 29:12315-12320.

Coghill RC, Sang CN, Maisog JM, Iadarola MJ (1999) Pain intensity processing within the human brain: a bilateral, distributed mechanism. J Neurophysiol 82:1934-1943.

Corbetta M, Shulman GL (2002) Control of goal-directed and stimulusdriven attention in the brain. Nat Rev Neurosci 3:201-215.

Cox RW (1996) AFNI: software for analysis and visualization of functional magnetic resonance neuroimages. Comput Biomed Res 29:162-173.

Craggs JG, Price DD, Perlstein WM, Nicholas Verne GN, Robinson ME (2008) The dynamic mechanisms of placebo induced analgesia: evidence of sustained and transient regional involvement. Pain 139:660-669.

Craig AD, Chen K, Bandy D, Reiman EM (2000) Thermosensory activation of insular cortex. Nat Neurosci 3:184-190.
Delgado MR, Li J, Schiller D, Phelps EA (2008) The role of the striatum in aversive learning and aversive prediction errors. Philos Trans R Soc Lond B Biol Sci 363:3787-3800.

Duann JR, Jung TP, Kuo WJ, Yeh TC, Makeig S, Hsieh JC, Sejnowski TJ (2002) Single-trial variability in event-related BOLD signals. Neuroimage 15:823-835.

Efron B, Tibshirani RJ (1993) An introduction to the bootstrap. New York: Chapman and Hall/CRC

Eickhoff SB, Paus T, Caspers S, Grosbras MH, Evans AC, Zilles K, Amunts K (2007) Assignment of functional activations to probabilistic cytoarchitectonic areas revisited. Neuroimage 36:511-521.

Eippert F, Bingel U, Schoell ED, Yacubian J, Klinger R, Lorenz J, Büchel C (2009) Activation of the opioidergic descending pain control system underlies placebo analgesia. Neuron 63:533-543.

Fanselow MS (1986) Conditioned fear-induced opiate analgesia: a competing motivational state theory of stress analgesia. Ann NY Acad Sci 467:40-54.

Fields H (2004) State-dependent opioid control of pain. Nat Rev Neurosci 5:565-575.

Fields HL (2007) Understanding how opioids contribute to reward and analgesia. Reg Anesth Pain Med 32:242-246.

Glover GH, Law CS (2001) Spiral-in/out BOLD fMRI for increased SNR and reduced susceptibility artifacts. Magn Reson Med 46:515-522.

Godinho F, Magnin M, Frot M, Perchet C, Garcia-Larrea L (2006) Emotional modulation of pain: is it the sensation or what we recall? J Neurosci 26:11454-11461.

Gottfried JA, O’Doherty J, Dolan RJ (2003) Encoding predictive reward value in human amygdala and orbitofrontal cortex. Science 301:1104-1107.

Graff-Guerrero A, González-Olvera J, Fresán A, Gómez-Martín D, MéndezNúñez JC, Pellicer F (2005) Repetitive transcranial magnetic stimulation of dorsolateral prefrontal cortex increases tolerance to human experimental pain. Brain Res Cogn Brain Res 25:153-160.

Hare TA, O’Doherty J, Camerer CF, Schultz W, Rangel A (2008) Dissociating the role of the orbitofrontal cortex and the striatum in the computation of goal values and prediction errors. J Neurosci 28:5623-5630.

Helmchen C, Mohr C, Roehl M, Bingel U, Lorenz J, Büchel C (2008) Common neural systems for contact heat and laser pain stimulation reveal higher-level pain processing. Hum Brain Mapp 29:1080-1091.

Holland PW (1988) Causal inference, path analysis, and recursive structural equations models. Sociol Methodol 18:449-484.

Jensen J, McIntosh AR, Crawley AP, Mikulis DJ, Remington G, Kapur S (2003) Direct activation of the ventral striatum in anticipation of aversive stimuli. Neuron 40:1251-1257.

Jensen J, Smith AJ, Willeit M, Crawley AP, Mikulis DJ, Vitcu I, Kapur S (2007) Separate brain regions code for salience vs. valence during reward prediction in humans. Hum Brain Mapp 28:294-302.

Kastner S, Pinsk MA, De Weerd P, Desimone R, Ungerleider LG (1999) Increased activity in human visual cortex during directed attention in the absence of visual stimulation. Neuron 22:751-761.

Keltner JR, Furst A, Fan C, Redfern R, Inglis B, Fields HL (2006) Isolating the modulatory effect of expectation on pain transmission: a functional magnetic resonance imaging study. J Neurosci 26:4437-4443.

Kenny DA, Korchmaros JD, Bolger N (2003) Lower level mediation in multilevel models. Psychol Methods 8:115-128.

Kirsch I (1985) Response expectancy as a determinant of experience and behavior. Am Psychol 40:1189-1202.

Knutson B, Adams CM, Fong GW, Hommer D (2001) Anticipation of increasing monetary reward selectively recruits nucleus accumbens. J Neurosci 21:RC159.

Kober H, Barrett L, Joseph J, Bliss-Moreau E, Lindquist K, Wager T (2008) Functional grouping and cortical-subcortical interactions in emotion: a meta-analysis of neuroimaging studies Neuroimage 42:998-1031.

Kong J, Gollub RL, Rosman IS, Webb JM, Vangel MG, Kirsch I, Kaptchuk TJ (2006) Brain activity associated with expectancy-enhanced placebo analgesia as measured by functional magnetic resonance imaging. J Neurosci 26:381-388.

Koyama T, McHaffie JG, Laurienti PJ, Coghill RC (2003) The single-epoch fMRI design: validation of a simplified paradigm for the collection of subjective ratings. Neuroimage 19:976-987.

Koyama T, McHaffie JG, Laurienti PJ, Coghill RC (2005) The subjective 
experience of pain: where expectations become reality. Proc Natl Acad Sci U S A 102:12950-12955.

Lefaucheur JP, Drouot X, Keravel Y, Nguyen JP (2001) Pain relief induced by repetitive transcranial magnetic stimulation of precentral cortex. Neuroreport 12:2963-2965.

Lieberman MD, Jarcho JM, Berman S, Naliboff BD, Suyenobu BY, Mandelkern M, Mayer EA (2004) The neural correlates of placebo effects: a disruption account. Neuroimage 22:447-455.

Lim SL, Padmala S, Pessoa L (2009) Segregating the significant from the mundane on a moment-to-moment basis via direct and indirect amygdala contributions. Proc Natl Acad Sci U S A:106:16841-16846.

Lindquist M, Meng Loh J, Atlas LY, Wager TD (2009) Modeling the hemodynamic response function in fMRI: efficiency, bias and mis-modeling. Neuroimage 45 [Suppl 1]:S187-S198.

Lorenz J, Hauck M, Paur RC, Nakamura Y, Zimmermann R, Bromm B, Engel AK (2005) Cortical correlates of false expectations during pain intensity judgments-a possible manifestation of placebo/nocebo cognitions. Brain Behav Immun 19:283-295.

MacKinnon DP, Warsi G, Dwyer JH (1995) A simulation study of mediated effect measures. Multivariate Behav Res 30:41.

MacKinnon DP, Krull JL, Lockwood CM (2000) Equivalence of the mediation, confounding and suppression effect. Prev Sci 1:173-181.

McNally GP (1999) Pain facilitatory circuits in the mammalian central nervous system: their behavioral significance and role in morphine analgesic tolerance. Neurosci Biobehav Rev 23:1059-1078.

Menon M, Jensen J, Vitcu I, Graff-Guerrero A, Crawley A, Smith MA, Kapur S (2007) Temporal difference modeling of the blood-oxygen level dependent response during aversive conditioning in humans: effects of dopaminergic modulation. Biol Psychiatry 62:765-772.

Mohr C, Binkofski F, Erdmann C, Büchel C, Helmchen C (2005) The anterior cingulate cortex contains distinct areas dissociating external from self-administered painful stimulation: a parametric fMRI study. Pain 114:347-357.

Moulton EA, Keaser ML, Gullapalli RP, Greenspan JD (2005) Regional intensive and temporal patterns of functional MRI activation distinguishing noxious and innocuous contact heat. J Neurophysiol 93:2183-2193.

Nahmias F, Debes C, de Andrade D, Mhalla A, Bouhassira D (2009) Diffuse analgesic effects of unilateral repetitive transcranial magnetic stimulation (rTMS) in healthy volunteers. Pain 147:224-232.

O'Doherty JP, Dayan P, Friston K, Critchley H, Dolan RJ (2003) Temporal difference models and reward-related learning in the human brain. Neuron 28:329-337.

Pessoa L (2009) How do emotion and motivation direct executive control? Trends Cogn Sci 13:160-166.

Petrovic P, Kalso E, Petersson KM, Ingvar M (2002) Placebo and opioid analgesia- imaging a shared neuronal network. Science 295:1737-1740.

Petrovic P, Dietrich T, Fransson P, Andersson J, Carlsson K, Ingvar M (2005) Placebo in emotional processing-induced expectations of anxiety relief activate a generalized modulatory network. Neuron 46:957-969.

Peyron R, Laurent B, García-Larrea L (2000) Functional imaging of brain responses to pain: a review and meta-analysis. Neurophysiol Clin 30:263-288.

Plassmann H, O’Doherty J, Shiv B, Rangel A (2008) Marketing actions can modulate neural representations of experienced pleasantness. Proc Natl Acad Sci U S A 105:1050-1054.

Ploner M, Lee MC, Wiech K, Bingel U, Tracey I (2010) Prestimulus functional connectivity determines pain perception in humans. Proc Natl Acad Sci U S A 107:355-360.

Price DD, Craggs J, Verne GN, Perlstein WM, Robinson ME (2007) Placebo analgesia is accompanied by large reductions in pain-related brain activity in irritable bowel syndrome patients. Pain 127:63-72.

Rescorla RA (1988) Behavioral studies of Pavlovian conditioning. Annu Rev Neurosci 11:329-352.

Rissman J, Gazzaley A, D’Esposito M (2004) Measuring functional connectivity during distinct stages of a cognitive task. Neuroimage 23:752-763.

Robinson ME, Gagnon CM, Riley JL 3rd, Price DD (2003) Altering gender role expectations: effects on pain tolerance, pain threshold, and pain ratings. J Pain 4:284-288.

Rolls ET, Grabenhorst F, Parris BA (2008) Warm pleasant feelings in the brain. Neuroimage 41:1504-1513.

Saddoris MP, Gallagher M, Schoenbaum G (2005) Rapid associative encod- ing in basolateral amygdala depends on connections with orbitofrontal cortex. Neuron 46:321-331.

Schnitzler A, Ploner M (2000) Neurophysiology and functional neuroanatomy of pain perception. J Clin Neurophysiol 17:592-603.

Schoenbaum G, Saddoris MP, Stalnaker TA (2007) Reconciling the roles of orbitofrontal cortex in reversal learning and the encoding of outcome expectancies. Ann NY Acad Sci 1121:320-335.

Scott DJ, Stohler CS, Egnatuk CM, Wang H, Koeppe RA, Zubieta JK (2007) Individual differences in reward responding explain Placebo-induced expectations and effects. Neuron 55:325-336.

Seymour B, O’Doherty JP, Koltzenburg M, Wiech K, Frackowiak R, Friston K, Dolan R (2005) Opponent appetitive-aversive neural processes underlie predictive learning of pain relief. Nat Neurosci 8:1234-1240.

Shattuck DW, Mirza M, Adisetiyo V, Hojatkashani C, Salamon G, Narr KL, Poldrack RA, Bilder RM, Toga AW (2008) Construction of a 3D probabilistic atlas of human cortical structures. Neuroimage 39:1064-1080.

Shrout PE, Bolger N (2002) Mediation in experimental and nonexperimental studies: New procedures and recommendations. Psychol Methods 7:422-445.

Singer T, Seymour B, O’Doherty J, Kaube H, Dolan RJ, Frith CD (2004) Empathy for pain involves the affective but not sensory components of pain. Science 303:1157-1162.

Sobel ME (2008) Identification of causal parameters in randomized studies with mediating variables. J Educ Behav Stat 33:230-251.

Stone CA, Sobel ME (1990) The robustness of estimates of total indirect effects in covariance structure models estimated by maximum likelihood. Psychometrika 55:337-352.

Tom SM, Fox CR, Trepel C, Poldrack RA (2007) The neural basis of loss aversion in decision-making under risk. Science 315:515-518.

Treede RD, Kenshalo DR, Gracely RH, Jones AK (1999) The cortical representation of pain. Pain 79:105-111.

Wager TD, Nichols TE (2003) Optimization of experimental design in fMRI: a general framework using a genetic algorithm. Neuroimage 18:293-309.

Wager TD, Rilling JK, Smith EE, Sokolik A, Casey KL, Davidson RJ, Kosslyn SM, Rose RM, Cohen JD (2004) Placebo-induced changes in FMRI in the anticipation and experience of pain. Science 303:1162-1167.

Wager TD, Keller MC, Lacey SC, Jonides J (2005) Increased sensitivity in neuroimaging analyses using robust regression. Neuroimage 26:99-113.

Wager TD, Lindquist M, Kaplan L (2007a) Meta-analysis of functional neuroimaging data: current and future directions. Soc Cogn Affect Neurosci 2:150-158.

Wager TD, Scott DJ, Zubieta JK (2007b) Placebo effects on human $\mu$-opioid activity during pain. Proc Natl Acad Sci U S A 104:11056-11061.

Wager TD, Davidson ML, Hughes BL, Lindquist MA, Ochsner KN (2008) Prefrontal-subcortical pathways mediating successful emotion regulation. Neuron: 59:1037-1050.

Wager TD, Waugh CE, Lindquist M, Noll DC, Fredrickson BL, Taylor SF (2009) Brain mediators of cardiovascular responses to social threat: part I: reciprocal dorsal and ventral sub-regions of the medial prefrontal cortex and heart-rate reactivity Neuroimage 47:821-835.

Weisenberg M, Schwarzwald J, Tepper I (1996) The influence of warning signal timing and cognitive preparation on the aversiveness of coldpressor pain. Pain 64:379-385.

Whalen PJ, Rauch SL, Etcoff NL, McInerney SC, Lee MB, Jenike MA (1998) Masked presentations of emotional facial expressions modulate amygdala activity without explicit knowledge. J Neurosci 18:411-418.

Wiech K, Kalisch R, Weiskopf N, Pleger B, Stephan KE, Dolan RJ (2006) Anterolateral prefrontal cortex mediates the analgesic effect of expected and perceived control over pain. J Neurosci 26:11501-11509.

Yang JC, Richlin D, Brand L, Wagner J, Clark WC (1985) Thermal sensory decision theory indices and pain threshold in chronic pain patients and healthy volunteers. Psychosom Med 47:461-468.

Zaki J, Ochsner KN, Hanelin J, Wager TD, Mackey SC (2007) Different circuits for different pain: patterns of functional connectivity reveal distinct networks for processing pain in self and others. Soc Neurosci 2:276-291.

Zubieta JK, Smith YR, Bueller JA, Xu Y, Kilbourn MR, Jewett DM, Meyer CR, Koeppe RA, Stohler CS (2001) Regional mu opioid receptor regulation of sensory and affective dimensions of pain. Science 293: 311-315. 